\title{
Measurement of the Triple-Gluon Vertex from 4-Jet Events at LEP
}

\author{
DELPHI Collaboration
}

\begin{abstract}
From the combined data of 1990 and 1991 of the DELPHI experiment at LEP, 13057 4-jet events are obtained and used for determining the contribution of the triple-gluon vertex. The relevant variables are the generalized Nachtmann Reiter angle $\theta_{N R}^{*}$ and the opening angle of the two least energetic jets. A fit to their two-dimensional distribution yields

$$
C_{A} / C_{F}=2.12 \pm 0.35 \text { and } \quad N_{C} / N_{A}=0.46 \pm 0.19
$$

where $C_{A} / C_{F}$ is the ratio of the coupling strength of the triple-gluon vertex to that of gluon bremsstrahlung from quarks, and $N_{C} / N_{A}$, the ratio of the number of quark colours to the number of gluons.

This constitutes a convincing model-independent proof of the existence of the triple-gluon vertex, since its contribution is directly proportional to $C_{A} / C_{F}$. The results are in agreement with the values expected from QCD: $C_{A} / C_{F}=$ 2.25 , and $N_{C} / N_{A}=3 / 8$.
\end{abstract}




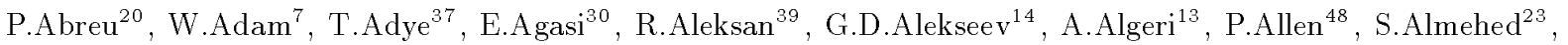
S.J.Alvsvaag ${ }^{4}$, U.Amaldi ${ }^{7}$, A.Andreazza ${ }^{27}$, P.Antilogus ${ }^{24}$, W-D.Apel ${ }^{15}$, R.J.Apsimon ${ }^{37}, \quad$ Y.Arnoud $^{39}$, B.Asman ${ }^{44}$, J-E.Augustin ${ }^{18}$, A.Augustinus ${ }^{30}$, P.Baillon ${ }^{7}$, P.Bambade ${ }^{18}$, F.Barao ${ }^{20}$, R.Barate ${ }^{12}$, G.Barbiellini ${ }^{46}$, D.Y.Bardin ${ }^{14}$, G.J.Barker ${ }^{34}$, A.Baroncelli ${ }^{40}$, O.Barring ${ }^{7}$, J.A.Barrio ${ }^{25}$, W.Bartl ${ }^{49}$, M.J.Bates ${ }^{37}$, M.Battaglia ${ }^{13}$, M.Baubillier ${ }^{22}$, K-H.Becks ${ }^{51}$, C.J.Beeston ${ }^{34}, \quad$ M.Begalli ${ }^{36}$, P.Beilliere ${ }^{6}$, Yu.Belokopytov ${ }^{42}$, P.Beltran ${ }^{9}$, D.Benedic $^{8}$, A.C.Benvenutit ${ }^{5}$, M.Berggren ${ }^{18}$, D.Bertrand ${ }^{2}$, F.Bianchi ${ }^{45}$, M.S.Bilenky ${ }^{14}$, P.Billoir ${ }^{22}$, J.Bjarne ${ }^{23}$,

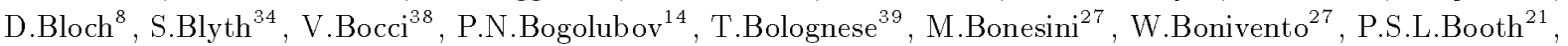
G.Borisov $^{42}$, H.Borner ${ }^{7}$, C.Bosio ${ }^{40}$, B.Bostjancic ${ }^{43}$, S.Bosworth $^{34}$, O.Botner ${ }^{47}$, B.Bouquet ${ }^{18}$, C.Bourdarios ${ }^{18}$, T.J.V.Bowcock ${ }^{21}$, M.Bozzo ${ }^{11}$, S.Braibant ${ }^{2}$, P.Branchini ${ }^{40}$, K.D.Brand ${ }^{35}$, R.A.Brenner ${ }^{7}$, H.Briand ${ }^{22}$, C.Bricman $^{2}$, R.C.A.Brown ${ }^{7}$, N.Brummer ${ }^{30}$, J-M.Brunet ${ }^{6}$, L.Bugge ${ }^{32}$, T.Buran ${ }^{32}$, H.Burmeister ${ }^{7}$, J.A.M.A.Buytaert ${ }^{7}$, M.Caccia ${ }^{7}$, M.Calvi ${ }^{27}$, A.J.Camacho Rozas ${ }^{41}$, R.Campion ${ }^{21}$, T.Camporesi ${ }^{7}$, V.Canale ${ }^{38}$, F.Cao ${ }^{2}$, F.Carena ${ }^{7}$, L.Carroll ${ }^{21}$, C.Caso ${ }^{11}$, M.V.Castillo Gimenez ${ }^{48}$, A.Cattai ${ }^{7}$, F.R.Cavallo ${ }^{5}$, L.Cerrito ${ }^{38}$, V.Chabaud ${ }^{7}$, A.Chan $^{1}$, M.Chapkin ${ }^{42}$, Ph.Charpentier ${ }^{7}$, L.Chaussard ${ }^{18}$, J.Chauveau ${ }^{22}$, P.Checchia ${ }^{35}$, G.A.Chelkov ${ }^{14}$, L.Chevalier ${ }^{39}$, P.Chliapnikov ${ }^{42}$, V.Chorowicz ${ }^{22}$, J.T.M.Chrin ${ }^{48}$, M.P.Clara ${ }^{45}$, P.Collins ${ }^{34}$, J.L.Contreras ${ }^{25}$, R.Contri ${ }^{11}$, E.Cortina ${ }^{48}$, G.Cosme ${ }^{18}$, F.Couchot ${ }^{18}$, H.B.Crawley ${ }^{1}$, D.Crennell ${ }^{37}$, G.Crosetti $^{11}$, M.Crozon $^{6}$, J.Cuevas Maestro ${ }^{33}$, S.Czellar ${ }^{13}$, E.Dahl-Jensen ${ }^{28}$, B.Dalmagne ${ }^{18}$, M.Dam ${ }^{32}$, G.Damgaard ${ }^{28}$, G.Darbo $^{11}$, E.Daubie ${ }^{2}$, A.Daum ${ }^{15}$, P.D.Dauncey ${ }^{34}$, M.Davenport ${ }^{7}$, P.David ${ }^{22}$, J.Davies ${ }^{21}$, W.Da Silva ${ }^{22}$, C.Defoix $^{6}$, P.Delpierre ${ }^{6}$, N.Demaria ${ }^{45}$, A.De Angelis ${ }^{46}$, H.De Boeck ${ }^{2}$, W.De Boer ${ }^{15}$, C.De Clercq ${ }^{2}$, M.D.M.De Fez Laso ${ }^{48}$, N.De Groot ${ }^{30}$, C.De La Vaissiere ${ }^{22}$, B.De Lotto ${ }^{46}$, A.De Min ${ }^{27}, \quad$ H.Dijkstra ${ }^{7}$, L.Di Ciaccio ${ }^{38}$, F.Djama ${ }^{8}$, J.Dolbeau ${ }^{6}$, M.Donszelmann $^{7}$, K.Doroba ${ }^{50}$, M.Dracos ${ }^{7}$, J.Drees ${ }^{51}$, M.Dris ${ }^{31}$, Y.Dufour $^{7}$, F.Dupont ${ }^{12}$, L-O.Eek ${ }^{47}$, P.A.-M.Eerola ${ }^{7}$, R.Ehret ${ }^{15}$, T.Ekelof ${ }^{47}$, G.Ekspong ${ }^{44}$, A.Elliot Peisert ${ }^{35}$, J-P.Engel $^{8}$, N.Ershaidat ${ }^{22}$, D.Fassouliotis ${ }^{31}$, M.Feindt ${ }^{7}$, M.Fernandez Alonso ${ }^{41}$, A.Ferrer ${ }^{48}$, T.A.Filippas ${ }^{31}$, A.Firestone $^{1}$, H.Foeth ${ }^{7}$, E.Fokitis ${ }^{31}$, F.Fontanelli ${ }^{11}$, K.A.J.Forbes ${ }^{21}$, J-L.Fousset ${ }^{26}$, S.Francon ${ }^{24}$, B.Franek ${ }^{37}$, P.Frenkiel $^{6}$, D.C.Fries ${ }^{15}$, A.G.Frodesen ${ }^{4}$, R.Fruhwirth ${ }^{49}$, F.Fulda-Quenzer ${ }^{18}$, K.Furnival ${ }^{21}$, H.Furstenau ${ }^{15}$, J.Fuster ${ }^{7}$, D.Gamba ${ }^{45}$, C.Garcia ${ }^{48}$, J.Garcia ${ }^{41}$, C.Gaspar ${ }^{7}$, U.Gasparini ${ }^{35}$, Ph.Gavillet ${ }^{7}$, E.N.Gazis ${ }^{31}$, J-P.Gerber $^{8}$, P.Giacomelli ${ }^{7}$, R.Gokieli ${ }^{50}$, B.Golob ${ }^{43}$, V.M.Golovatyuk ${ }^{14}$, J.J.Gomez Y Cadenas ${ }^{7}$, A.Goobar ${ }^{44}$, G.Gopal $^{37}$, M.Gorski ${ }^{50}$, V.Gracco ${ }^{11}$, A.Grant ${ }^{7}$, F.Grard ${ }^{2}$, E.Graziani ${ }^{40}$, G.Grosdidier ${ }^{18}$, E.Gross ${ }^{7}$, P.Grosse-Wiesmann ${ }^{7}$, B.Grossetete ${ }^{22}$, J.Guy ${ }^{37}$, U.Haedinger ${ }^{15}$, F.Hahn ${ }^{51}$, M.Hahn ${ }^{15}$, S.Haider ${ }^{30}$, A.Hakansson $^{23}$, A.Hallgren ${ }^{47}$, M.Halpaap ${ }^{15}$, K.Hamacher ${ }^{51}$, G.Hamel De Monchenault ${ }^{39}$, W.Hao ${ }^{30}$, F.J.Harris ${ }^{34}$, V.Hedberg ${ }^{23}$, T.Henkes ${ }^{7}$, J.J.Hernandez ${ }^{48}$, P.Herquet ${ }^{2}$, H.Herr ${ }^{7}$, T.L.Hessing ${ }^{21}$, I.Hietanen ${ }^{13}$, C.O.Higgins ${ }^{21}$, E.Higon ${ }^{48}$, H.J.Hilke ${ }^{7}$, S.D.Hodgson ${ }^{34}$, T.Hofmokl ${ }^{50}$, R.Holmes ${ }^{1}, \quad$ S-O.Holmgren ${ }^{44}$, D.Holthuizen $^{30}$, P.F.Honore ${ }^{6}$, J.E.Hooper ${ }^{28}$, M.Houlden ${ }^{21}$, J.Hrubec ${ }^{49}$, K.Huet ${ }^{2}$, P.O.Hulth ${ }^{44}$, K.Hultqvist ${ }^{44}$, P.Ioannou $^{3}$, P-S.Iversen ${ }^{4}$, J.N.Jackson ${ }^{21}, \quad$ P.Jalocha ${ }^{16}, \quad$ G.Jarlskog ${ }^{23}, \quad$ P.Jarry ${ }^{39}$, B.Jean-Marie ${ }^{18}$, E.K.Johansson ${ }^{44}$, D.Johnson ${ }^{21}$, M.Jonker ${ }^{7}$, L.Jonsson ${ }^{23}$, P.Juillot ${ }^{8}$, G.Kalkanis ${ }^{3}$, G.Kalmus ${ }^{37}$, F.Kapusta ${ }^{22}$, M.Karlsson ${ }^{7}$, E.Karvelas ${ }^{9}$, S.Katsanevas ${ }^{3}$, E.C.Katsoufis ${ }^{31}$, R.Keranen ${ }^{7}$, J.Kesteman ${ }^{2}$, B.A.Khomenko ${ }^{14}$, N.N.Khovanski ${ }^{14}$, B.King ${ }^{21}$, N.J.Kjaer ${ }^{7}$, H.Klein ${ }^{7}$, A.Klovning ${ }^{4}$, P.Kluit ${ }^{30}$, A.Koch-Mehrin ${ }^{51}$, J.H.Koehne ${ }^{15}$, B.Koene $^{30}$, P.Kokkinias ${ }^{9}$, M.Koratzinos ${ }^{32}$, A.V.Korytov ${ }^{14}$, V.Kostioukhine ${ }^{42}$, C.Kourkoumelis ${ }^{3}$, O.Kouznetsov ${ }^{14}$, P.H.Kramer ${ }^{51}$, J.Krolikowski ${ }^{50}$, I.Kronkvist ${ }^{23}$, U.Kruener-Marquis ${ }^{51}$, W.Krupinski ${ }^{16}$, K.Kulka ${ }^{47}$, K.Kurvinen ${ }^{13}$, C.Lacasta ${ }^{48}$, C.Lambropoulos ${ }^{9}$, J.W.Lamsa ${ }^{1}$, L.Lanceri ${ }^{46}$, V.Lapin ${ }^{42}$, J-P.Laugier ${ }^{39}$, R.Lauhakangas $^{13}$, G.Leder ${ }^{49}$, F.Ledroit ${ }^{12}$, R.Leitner ${ }^{29}$, Y.Lemoigne ${ }^{39}$, J.Lemonne ${ }^{2}$, G.Lenzen ${ }^{\text {51 }}$, V.Lepeltier ${ }^{18}$,

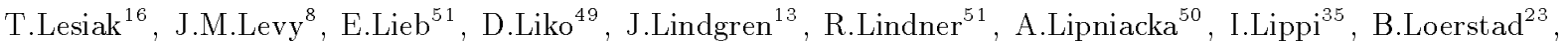
M.Lokajicek ${ }^{10}$, J.G.Loken ${ }^{34}$, A.Lopez-Fernandez ${ }^{7}$, M.A.Lopez Aguera ${ }^{41}$, M.Los ${ }^{30}$, D.Loukas ${ }^{9}$, J.J.Lozano ${ }^{48}$,

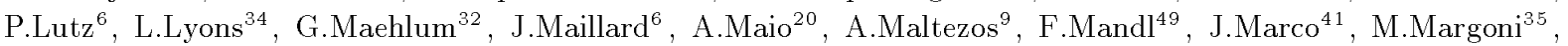
J-C.Marin ${ }^{7}$, A.Markou ${ }^{9}$, T.Maron ${ }^{51}$, S.Marti ${ }^{48}$, L.Mathis ${ }^{1}$, F.Matorras ${ }^{41}$, C.Matteuzzi ${ }^{27}$, G.Matthiae $^{38}$, M.Mazzucato $^{35}$, M.Mc Cubbin ${ }^{21}$, R.Mc Kay ${ }^{1}$, R.Mc Nulty ${ }^{21}$, G.Meola ${ }^{11}$, C.Meroni ${ }^{27}$, W.T.Meyer ${ }^{1}$, M.Michelotto $^{35}$, I.Mikulec ${ }^{49}$, L.Mirabito ${ }^{24}$, W.A.Mitaroff ${ }^{49}$, G.V.Mitselmakher ${ }^{14}$, U.Mjoernmark ${ }^{23}$, T.Moa ${ }^{44}$, R.Moeller $^{28}$, K.Moenig ${ }^{7}$, M.R.Monge ${ }^{11}$, P.Morettini ${ }^{11}$, H.Mueller ${ }^{15}$, W.J.Murray ${ }^{37}$, B.Muryn ${ }^{16}$, G.Myatt ${ }^{34}$, F.L.Navarria ${ }^{5}$, P.Negri ${ }^{27}$, R.Nicolaidou ${ }^{3}$, B.S.Nielsen ${ }^{28}$, B.Nijjhar ${ }^{21}$, V.Nikolaenko ${ }^{42}$, P.E.S.Nilsen ${ }^{4}$, P.Niss ${ }^{44}$, V.Obraztsov ${ }^{42}$, A.G.Olshevski ${ }^{14}$, R.Orava ${ }^{13}$, A.Ostankov ${ }^{42}$, K.Osterberg ${ }^{13}$, A.Ouraou ${ }^{39}$, M.Paganoni ${ }^{27}$, R.Pain ${ }^{22}$, H.Palka ${ }^{16}$, Th.D.Papadopoulou ${ }^{31}$, L.Pape ${ }^{7}$, F.Parodi ${ }^{11}$, A.Passeri ${ }^{40}$, M.Pegoraro ${ }^{35}$, J.Pennanen ${ }^{13}$, L.Peralta $^{20}$, V.Perevozchikov ${ }^{42}$, H.Pernegger ${ }^{49}$, M.Pernicka ${ }^{49}$, A.Perrotta ${ }^{5}$, C.Petridou ${ }^{46}$, A.Petrolini ${ }^{11}$, T.E.Pettersen ${ }^{35}$, F.Pierre ${ }^{39}$, M.Pimenta ${ }^{20}$, O.Pingot ${ }^{2}$, S.Plaszczynski ${ }^{18}$, O.Podobrin ${ }^{15}$, M.E.Pol ${ }^{7}$, G.Polok ${ }^{16}$, P.Poropat $^{46}$, P.Privitera ${ }^{15}$, A.Pullia ${ }^{27}$, D.Radojicic ${ }^{34}$, S.Ragazzi ${ }^{27}$, H.Rahmani ${ }^{31}$, P.N.Ratoff ${ }^{19}$, A.L.Read ${ }^{32}$, P.Rebecci $^{7}$, N.G.Redaelli ${ }^{27}$, M.Regler ${ }^{49}$, D.Reid ${ }^{7}$, P.B.Renton ${ }^{34}$, L.K.Resvanis ${ }^{3}$, F.Richard ${ }^{18}$, M.Richardson ${ }^{21}$, J.Ridky $^{10}$, G.Rinaudo ${ }^{45}$, I.Roditi ${ }^{17}$, A.Romero ${ }^{45}$, I.Roncagliolo ${ }^{11}$, P.Ronchese ${ }^{35}$, C.Ronnqvist ${ }^{13}$, E.I.Rosenberg ${ }^{1}$, S.Rossi $^{7}$, U.Rossi ${ }^{5}$, E.Rosso ${ }^{7}$, P.Roudeau $^{18}$, T.Rovelli $^{5}$, W.Ruckstuhl ${ }^{30}$, V.Ruhlmann-Kleider ${ }^{39}$, A.Ruiz $^{41}$, K.Rybicki ${ }^{16}$, H.Saarikko ${ }^{13}$, Y.Sacquin ${ }^{39}$, G.Sajot ${ }^{12}$, J.Salt ${ }^{48}$, J.Sanchez ${ }^{25}$, M.Sannino ${ }^{11}$, S.Schael ${ }^{7}$, H.Schneider ${ }^{15}$, B.Schulze ${ }^{38}$, M.A.E.Schyns ${ }^{51}$, G.Sciolla ${ }^{45}$, F.Scuri ${ }^{46}$, A.M.Segar ${ }^{34}$, A.Seitz ${ }^{15}$, R.Sekulin ${ }^{37}$, 
M.Sessa ${ }^{46}$, G.Sette ${ }^{11}$, R.Seufert ${ }^{15}$, R.C.Shellard ${ }^{36}$, I.Siccama ${ }^{30}$, P.Siegrist ${ }^{39}$, S.Simonetti ${ }^{11}$, F.Simonetto ${ }^{35}$, A.N.Sisakian ${ }^{14}$, G.Skjevling ${ }^{32}$, G.Smadja ${ }^{3924}$, N.Smirnov ${ }^{42}$, G.R.Smith ${ }^{37}$, R.Sosnowski ${ }^{50}$, D.Souza-Santos ${ }^{36}$, T.S.Spassoff ${ }^{12}$, E.Spiriti ${ }^{40}$, S.Squarcia ${ }^{11}$, H.Staeck ${ }^{51}$, C.Stanescu ${ }^{40}$, S.Stapnes ${ }^{32}$, G.Stavropoulos ${ }^{9}$, F.Stichelbaut $^{2}$, A.Stocchi ${ }^{18}$, J.Strauss ${ }^{49}$, J.Straver $^{7}$, R.Strub ${ }^{8}$, B.Stugu ${ }^{4}$, M.Szczekowski $^{7}$, M.Szeptycka ${ }^{50}$, P.Szymanski ${ }^{50}$, T.Tabarelli ${ }^{27}$, O.Tchikilev ${ }^{42}$, G.E.Theodosiou ${ }^{9}$, A.Tilquin ${ }^{26}$, J.Timmermans ${ }^{30}$, V.G.Timofeev ${ }^{14}$, L.G.Tkatchev ${ }^{14}$, T.Todorov ${ }^{8}$, D.Z.Toet ${ }^{30}$, O.Toker ${ }^{13}$, B.Tome ${ }^{20}$, E.Torassa ${ }^{45}$, L.Tortora ${ }^{40}$, D.Treille ${ }^{7}$, U.Trevisan $^{11}$, W.Trischuk ${ }^{7}$, G.Tristram ${ }^{6}$, C.Troncon ${ }^{27}$, A.Tsirou ${ }^{7}$, E.N.Tsyganov ${ }^{14}$, M.Turala ${ }^{16}$, M-L.Turluer ${ }^{39}$, T.Tuuva $^{13}$, I.A.Tyapkin ${ }^{22}$, M.Tyndel $^{37}$, S.Tzamarias ${ }^{21}$, S.Ueberschaer ${ }^{51}$, O.Ullaland ${ }^{7}$, V.Uvarov ${ }^{42}$, G.Valenti $^{5}$, E.Vallazza $^{45}$, J.A.Valls Ferrer ${ }^{48}$, C.Vander Velde ${ }^{2}$, G.W.Van Apeldoorn ${ }^{30}$, P.Van Dam ${ }^{30}$, M.Van Der Heijden ${ }^{30}$, W.K.Van Doninck ${ }^{2}$, P.Vaz ${ }^{7}$, G.Vegni $^{27}$, L.Ventura ${ }^{35}$, W.Venus ${ }^{37}$, F.Verbeure ${ }^{2}$, M.Verlato ${ }^{35}$, L.S.Vertogradov ${ }^{14}$, D.Vilanova ${ }^{39}$, P.Vincent ${ }^{24}$, L.Vitale ${ }^{13}$, E.Vlasov ${ }^{42}$, A.S.Vodopyanov ${ }^{14}$, M.Vollmer $^{51}$, M.Voutilainen $^{13}$, V.Vrba $^{40}$, H.Wahlen ${ }^{51}$, C.Walck ${ }^{44}$, F.Waldner ${ }^{46}$, M.Wayne ${ }^{1}$, A.Wehr ${ }^{51}$, M.Weierstall ${ }^{51}$, P.Weilhammer ${ }^{7}$, J.Werner ${ }^{51}$, A.M.Wetherell $^{7}$, J.H.Wickens ${ }^{2}$, G.R.Wilkinson ${ }^{34}$, W.S.C.Williams ${ }^{34}$, M.Winter ${ }^{8}$, M.Witek ${ }^{16}$, G.Wormser ${ }^{18}$, K.Woschnagg ${ }^{47}$, N.Yamdagni ${ }^{44}$, P.Yepes ${ }^{7}$, A.Zaitsev $^{42}$, A.Zalewska ${ }^{16}, \quad$ P.Zalewski ${ }^{18}$, D.Zavrtanik ${ }^{43}$, E.Zevgolatakos ${ }^{9}$, G.Zhang ${ }^{51}$, N.I.Zimin ${ }^{14}$, M.Zito ${ }^{39}$, R.Zuberi ${ }^{34}$, R.Zukanovich Funchal ${ }^{6}$, G.Zumerle ${ }^{35}$, J.Zuniga $^{48}$

\footnotetext{
${ }^{1}$ Ames Laboratory and Department of Physics, Iowa State University, Ames IA 50011, USA

${ }^{2}$ Physics Department, Univ. Instelling Antwerpen, Universiteitsplein 1, B-2610 Wilrijk, Belgium and IIHE, ULB-VUB, Pleinlaan 2, B-1050 Brussels, Belgium

and Faculté des Sciences, Univ. de l'Etat Mons, Av. Maistriau 19, B-7000 Mons, Belgium

${ }^{3}$ Physics Laboratory, University of Athens, Solonos Str. 104, GR-10680 Athens, Greece

${ }^{4}$ Department of Physics, University of Bergen, Allégaten 55, N-5007 Bergen, Norway

${ }^{5}$ Dipartimento di Fisica, Università di Bologna and INFN, Via Irnerio 46, I-40126 Bologna, Italy

${ }^{6}$ Collège de France, Lab. de Physique Corpusculaire, IN2P3-CNRS, F-75231 Paris Cedex 05, France

${ }^{7}$ CERN, CH-1211 Geneva 23, Switzerland

${ }^{8}$ Centre de Recherche Nucléaire, IN2P3 - CNRS/ULP - BP20, F-67037 Strasbourg Cedex, France

${ }^{9}$ Institute of Nuclear Physics, N.C.S.R. Demokritos, P.O. Box 60228, GR-15310 Athens, Greece

${ }^{10}$ FZU, Inst. of Physics of the C.A.S. High Energy Physics Division, Na Slovance 2, CS-180 40, Praha 8 , Czechoslovakia

${ }^{11}$ Dipartimento di Fisica, Università di Genova and INFN, Via Dodecaneso 33, I-16146 Genova, Italy

${ }^{12}$ Institut des Sciences Nucléaires, IN2P3-CNRS, Université de Grenoble 1, F-38026 Grenoble, France

${ }^{13}$ Research Institute for High Energy Physics, SEFT, Siltavuorenpenger 20 C, SF-00170 Helsinki, Finland

${ }^{14}$ Joint Institute for Nuclear Research, Dubna, Head Post Office, P.O. Box 79, 101000 Moscow, Russian Federation

${ }^{15}$ Institut für Experimentelle Kernphysik, Universität Karlsruhe, Postfach 6980, D-7500 Karlsruhe 1, Germany

${ }^{16}$ High Energy Physics Laboratory, Institute of Nuclear Physics, Ul. Kawiory 26 a, PL-30055 Krakow 30 , Poland

${ }^{17}$ Centro Brasileiro de Pesquisas Físicas, rua Xavier Sigaud 150, RJ-22290 Rio de Janeiro, Brazil

${ }^{18}$ Université de Paris-Sud, Lab. de l'Accélérateur Linéaire, IN2P3-CNRS, Bat 200, F-91405 Orsay, France

${ }^{19}$ School of Physics and Materials, University of Lancaster, GB - Lancaster LA1 4YB, UK

${ }^{20}$ LIP, IST, FCUL - Av. Elias Garcia, $14-1^{\circ}$, P-1000 Lisboa Codex, Portugal

${ }^{21}$ Department of Physics, University of Liverpool, P.O. Box 147, GB - Liverpool L69 3BX. UK

${ }^{22}$ LPNHE, IN2P3-CNRS, Universités Paris VI et VII, Tour 33 (RdC), 4 place Jussieu, F-75252 Paris Cedex 05, France

${ }^{23}$ Department of Physics, University of Lund, Sölvegatan 14, S-22363 Lund, Sweden

${ }^{24}$ Université Claude Bernard de Lyon, IPNL, IN2P3-CNRS, F-69622 Villeurbanne Cedex, France

${ }^{25}$ Universidad Complutense, Avda. Complutense s/n, E-28040 Madrid, Spain

${ }^{26}$ Univ. d'Aix - Marseille II - CPP, IN2P3-CNRS, F-13288 Marseille Cedex 09, France

${ }^{27}$ Dipartimento di Fisica, Università di Milano and INFN, Via Celoria 16, I-20133 Milan, Italy

${ }^{28}$ Niels Bohr Institute, Blegdamsvej 17, DK-2100 Copenhagen 0, Denmark

${ }^{29}$ NC, Nuclear Centre of MFF, Charles University, Areal MFF, V Holesovickach 2, CS-180 00, Praha 8, Czechoslovakia ${ }^{30}$ NIKHEF-H, Postbus 41882, NL-1009 DB Amsterdam, The Netherlands

${ }^{31}$ National Technical University, Physics Department, Zografou Campus, GR-15773 Athens, Greece

${ }^{32}$ Physics Department, University of Oslo, Blindern, N-1000 Oslo 3, Norway

33 Dpto. Fisica, Univ. Oviedo, C/P.Jimenez Casas, S/N-33006 Oviedo, Spain

${ }^{34}$ Nuclear Physics Laboratory, University of Oxford, Keble Road, GB - Oxford OX1 3RH, UK

${ }^{35}$ Dipartimento di Fisica. Università di Padova and INFN, Via Marzolo 8, I-35131 Padua, Italy

${ }^{36}$ Depto. de Fisica, Pontificia Univ. Católica, C.P. 38071 RJ-22453 Rio de Janeiro, Brazil

${ }^{37}$ Rutherford Appleton Laboratory, Chilton, GB - Didcot OX11 OQX, UK

${ }^{38}$ Dipartimento di Fisica, Università di Roma II and INFN, Tor Vergata, I-00173 Rome, Italy

${ }^{39}$ Centre d'Etude de Saclay, DSM/DAPNIA, F-91191 Gif-sur-Yvette Cedex, France

${ }^{40}$ Istituto Superiore di Sanità, Ist. Naz. di Fisica Nucl. (INFN), Viale Regina Elena 299, I-00161 Rome, Italy

${ }^{41}$ C.E.A.F.M., C.S.I.C. - Univ. Cantabria, Avda. los Castros, S/N-39006 Santander, Spain

${ }^{42}$ Inst. for High Energy Physics, Serpukow P.O. Box 35, Protvino, (Moscow Region), Russian Federation

43 J. Stefan Institute and Department of Physics, University of Ljubljana, Jamova 39, SI-61000 Ljubljana, Slovenia

${ }^{44}$ Institute of Physics, University of Stockholm, Vanadisvägen 9, S-113 46 Stockholm, Sweden

${ }^{45}$ Dipartimento di Fisica Sperimentale, Università di Torino and INFN, Via P. Giuria 1, I-10125 Turin, Italy

${ }^{46}$ Dipartimento di Fisica. Università di Trieste and INFN, Via A. Valerio 2, I-34127 Trieste, Italy

and Istituto di Fisica, Università di Udine, I-33100 Udine, Italy

${ }^{47}$ Department of Radiation Sciences, University of Uppsala, P.O. Box 535, S-751 21 Uppsala, Sweden

${ }^{48}$ IFIC, Valencia-CSIC, and D.F.A.M.N., U. de Valencia, Avda. Dr. Moliner 50, E-46100 Burjassot (Valencia), Spain

${ }^{49}$ Institut für Hochenergiephysik, Österr. Akad. d. Wissensch., Nikolsdorfergasse 18, A-1050 Vienna, Austria

50 Inst. Nuclear Studies and, University of Warsaw, Ul. Hoza 69, PL-00681 Warsaw, Poland

${ }^{51}$ Fachbereich Physik, University of Wuppertal, Postfach 100 127, D-5600 Wuppertal 1, Germany
} 


\section{Introduction}

An essential feature of Quantum Chromodynamics (QCD) is the self-coupling of the gluons due to their colour charges. The 'triple-gluon vertex' is a direct consequence of the non-Abelian nature of this gauge theory. The large two-jet rate for medium jet energies at hadron colliders can be considered as evidence for gluon-gluon scattering [1], if one accepts the extrapolation of the gluon structure function of the proton from deepinelastic $\nu N$-scattering to collider energies. A colourless gluon would lead to the reaction $\Upsilon \rightarrow 2$ jets [2] which is not observed [3]. In $e^{+} e^{-}$annihilation the energy dependence [4] of the strong coupling constant $\alpha_{s}$, where the triple gluon vertex enters through loop corrections, constitutes further indirect evidence. Direct evidence can be obtained from the study of 4-jet events in $e^{+} e^{-}$annihilation, as in 4-parton final states the triple-gluon vertex contributes to the Born diagrams.

Several observables have been proposed [5-8] to test the presence of the triple-gluon vertex experimentally. In the study of the angular distributions of 4-jet events, several collaborations [10-13] have published evidence against a QED-like Abelian theory [5,14] of the strong interaction in which the gluon is colourless but the threefold colour of the quarks is retained. The Abelian theory is disproved by its much higher secondary $q \bar{q}-$ production, since the distributions considered distinguish well between the contribution from secondary quark-antiquark production (fig. 1b) and that from two gluons (figs. 1a, 1c), due to their different helicity structures. More generally two experiments $[11,13]$ have provided limits on the relative contribution from secondary $q \bar{q}$-production. However, these results do not give evidence for the existence of the triple-gluon vertex since the one-dimensional angular distributions studied do not distinguish between the contribution from the triple-gluon vertex (fig. 1c) and double-bremsstrahlung (fig. 1a).

In a previous analysis of 4-jet events [15] two-dimensional distributions have been studied. As in the other publications the generalized Nachtmann-Reiter angle $\theta_{N R}^{*}$ (fig. 2) [9] has been used since it distinguishes between two-gluon final states and secondary $q \bar{q}$-production. It is defined as the angle between $\left(\vec{p}_{1}-\vec{p}_{2}\right)$ and $\left(\vec{p}_{3}-\vec{p}_{4}\right)$, where the $\vec{p}_{i}$ are the 3 -vectors of the four jets in decreasing energy order. The additional observable, the angle between the two least energetic jets $\alpha_{34}$ (fig. 2), distinguishes between triple-gluon vertex and double-bremsstrahlung. The contribution from the triple-gluon vertex may be determined directly from the two-dimensional distribution of these observables.

The initial analysis suffered from the limited statistics of the full simulation of the detector. This induced a bias in the result, due to the lack of smoothness in the reference distributions for the different 4-parton final states. This bias was estimated and corrected for, but was an additional source of uncertainty. In the present paper the analysis is extended to all data of 1990 and 1991. Smooth reference distributions were produced by generating very large event samples. Full simulation of the DELPHI detector is needed only for an overall correction of detector effects.

\section{Theoretical Basis}

The triple-gluon vertex in $e^{+} e^{-}$annihilation appears in terms which are second order and higher in the strong coupling constant. The principal second order contributions to the 4-parton final states are: double-bremsstrahlung, triple-gluon vertex and secondary 
$q \bar{q}$-production. The diagrams are shown in fig. 1. Thus testing the triple-gluon vertex requires a study of 4-jet events. With the four jets ordered according to energy, jet 3 and jet 4 correspond preferentially to the secondary partons.

The fundamental couplings are illustrated in fig. 3. The Casimir factors $C_{F}, C_{A}$, $T_{F}$ are respectively a measure the coupling strengths of gluon radiation from quarks, of the triple-gluon vertex, and of gluon splitting into a quark-antiquark pair. For any representation of a gauge group describing these couplings, they are determined in terms of its generators $t_{a b}^{r}$ and its structure constants $f^{r s t}$ by the relations (the notation of $\mathrm{T}$. Hebbeker [16] is used):

$$
\begin{aligned}
t_{a b}^{r} t_{b c}^{r} & =\delta_{a c} C_{F} \\
f^{r s t} f^{r s u} & =\delta^{t u} C_{A} \\
t_{a b}^{r} t_{b a}^{s} & =\delta_{r s} T_{F}
\end{aligned}
$$

where:

$$
\begin{aligned}
a, b, \ldots= & 1, \ldots, N_{C} \quad \text { quark color index } \\
r, s, \ldots= & 1, \ldots, N_{A} \quad \text { gluon color index }
\end{aligned}
$$

and repeated indices are to be summed. [17]:

The ratio of the coupling strength $T_{F}$ for $g \rightarrow q \bar{q}$ to $C_{F}$ for $q \rightarrow q g$ is then given by

$$
T_{F} / C_{F}=N_{C} / N_{A}
$$

The interference terms contain combinations of these basic couplings and this leads to more complicated graphs for the transition probabilities. The graphs can be grouped as simple planar ones and the more complicated nonplanar graphs where particle lines cross. R.K. Ellis et al. [18] have calculated the differential cross sections for the production of the 4-parton final states in order $\alpha_{s}^{2}$. In figs. 6 and 8 of their paper, all topologically distinct graphs for the transition probabilities are shown. Second order Matrix Elements (ME method) should provide a more reliable representation of hard jets for the analysis of the triple-gluon vertex than the Parton Shower models (PS) (see M. Bengtsson [19]. The two approaches are also discussed by S. Bethke et al. [20].) For the $q \bar{q} g g$ final state there are 36 contributions which can be grouped into three classes:

A: planar double-bremsstrahlung graphs with weight $C_{F}^{2}$;

B: non-planar double-bremsstrahlung graphs with weight $C_{F}\left(C_{F}-\frac{1}{2} C_{A}\right)$;

$\mathrm{C}$ : graphs involving the triple-gluon vertex with weight $C_{F} C_{A}$.

Similarily the 36 contributions for $q \bar{q} q \bar{q}$ fall into the classes:

D: planar graphs with weight $C_{F} T_{R}$;

E: non-planar graphs with weight $C_{F}\left(C_{F}-\frac{1}{2} C_{A}\right)$;

$\mathrm{F}$ : graphs with weight $C_{F}$, which give contributions only if the charge of the partons is determined experimentally and are therefore not relevant to this analysis.

$T_{R}$ and $T_{F}$ are related by

$$
T_{R}=T_{F} n_{f}
$$


where $n_{f}$ is the number of active quark flavours.

The variable $N_{C}$ originally appearing in these expressions has been replaced by $C_{A}$, as the really relevant coupling is that for the triple-gluon vertex [17]. In $\mathrm{SU}\left(N_{C}\right)$ gauge theory and in particular in QCD, the quantities $C_{A}$ and $N_{C}$ are equal. In other gauge groups however the different physical meaning of these factors results in different numerical values.

The differential cross section for 4-jet production in $e^{+} e^{-}$annihilation can be written in the form:

$$
\sigma_{4}\left(y_{i j}\right)=\sigma_{q \bar{q} g g}\left(y_{i j}\right)+\sigma_{q \bar{q} q \bar{q}}\left(y_{i j}\right)
$$

Here $y_{i j}=m_{i j}^{2} / s \quad(i, j=1, . ., 4)$, and denote the effective masses squared for any pair of jets (partons); and

$$
\begin{aligned}
\sigma_{q \bar{q} g g} & =\sigma_{0}\left[F_{A}\left(y_{i j}\right)+\left(1-\frac{1}{2} \frac{\mathbf{C}_{\mathbf{A}}}{\mathbf{C}_{\mathbf{F}}}\right) F_{B}\left(y_{i j}\right)+\frac{\mathbf{C}_{\mathbf{A}}}{\mathbf{C}_{\mathbf{F}}} F_{C}\left(y_{i j}\right)\right] \\
\sigma_{q \bar{q} q \bar{q}} & =\sigma_{0}\left[\frac{\mathbf{T}_{\mathbf{R}}}{\mathbf{C}_{\mathbf{F}}} F_{D}\left(y_{i j}\right)+\left(1-\frac{1}{2} \frac{\mathbf{C}_{\mathbf{A}}}{\mathbf{C}_{\mathbf{F}}}\right) F_{E}\left(y_{i j}\right)\right]
\end{aligned}
$$

where $\sigma_{0}$ is the zeroth order 2-parton cross section given with the quark charges $e_{k}$ by

$$
\sigma_{0}=\frac{4 \pi \alpha^{2}}{3 s} N_{C} \sum_{k=1}^{n_{f}} e_{k}^{2}
$$

The analytic form of the kinematical functions $F_{A}, \ldots, F_{E}$ can be derived from the formulae in ref. [18]. For QCD the fermionic Casimir operator is $C_{F}=\frac{4}{3}$, the coupling strength of the triple-gluon vertex $C_{A}=3$ and $T_{F}=\frac{1}{2}$. For the Abelian theory the values are $C_{F}=1, C_{A}=0, T_{F}=3$ and for QED $C_{F}=1, C_{A}=0, T_{F}=1$. The values for $N_{C} / N_{A}$ and $C_{A} / C_{F}$ in other gauge groups are given in table 1 [17]. Since the grouping of the graphs is done in a gauge invariant way, one can determine the contributions from these classes, and use their relative weights as a test of QCD and compare also with the predictions of other gauge groups.

In this analysis the contributions of the classes are considered as functions of two observables. One is as usual the generalised Nachtmann-Reiter angle $\theta_{N R}^{*}$. The second observable is the opening angle $\alpha_{34}$ of the jets from the secondary partons. These observables have the advantage that no cuts in opening angles are needed on the 4-jet sample. The two observables of our analysis are illustrated in fig. 2. There is some correlation between $\theta_{N R}^{*}$ and $\alpha_{34}$; the study is therefore performed by plotting the two-dimensional distribution in the angular observables. Two-dimensional reference distributions in the observables are provided for the five classes by Monte Carlo simulation. From the reference distributions in $\left|\cos \theta_{N R}^{*}\right|$ and $\cos \alpha_{34}$, the expected two-dimensional distribution is expressed as a function of $C_{A} / C_{F}$ and $T_{R} / C_{F}$. The variables $C_{A} / C_{F}$ and $T_{R} / C_{F}$ are then determined in a fit of this distribution to the corresponding one of the data, corrected for effects of the detector. 


\section{Treatment of Data}

This analysis is based on the data of 1990 and 1991 from $e^{+} e^{-}$annihilations at c.m.s. energies around the $Z^{0}$ resonance. Only tracks from charged particles are used. The same cuts are applied for event selection as in the earlier study [21] of hadronic decays of the $Z^{0}$. The most important of these selections are that tracks are kept only if the measured track length is greater than $50 \mathrm{~cm}$ and their polar angle is between $25^{\circ}$ and $155^{\circ}$. Furthermore, for all events the polar angle $\theta$ of the sphericity axis has to be between $40^{\circ}$ and $140^{\circ}$ and the total momentum imbalance below $20 \mathrm{GeV} / \mathrm{c}$. After the selections 68862 and 154424 multihadron events remained.

Jets of charged particles are determined with the algorithm LUCLUS provided with the LUND Monte Carlo program [23], called JETSET. In this algorithm two jets with 3 -momenta $p_{1}, p_{2}$ and opening angle $\alpha_{12}$ are merged if $2 \frac{p_{1} * p_{2}}{p_{1}+p_{2}} * \sin \frac{\alpha_{12}}{2} \leq d_{\text {join }}$. Each time two jets are merged, new jet axes are determined and all particles are reassigned to the nearest jet. With the new jets so defined, the procedure is repeated until a stable configuration is reached. The jet resolution parameter $d_{\text {join }}$ is set to a fixed value of 3 $\mathrm{GeV}$. This corresponds to a value of $m_{\text {eff }}$ of any two jets of at least $9.8 \mathrm{GeV}$ when scaled with the ratio of $E_{c m s}$ and the average charged visible energy of $55 \mathrm{GeV}$. It is required that $E_{\text {vis }}>0.20 E_{\mathrm{cms}}$, where $E_{\text {vis }}$ is the sum of the energies of the accepted charged particles, and $E_{c m s}=\sqrt{s}$. This yields 4279 and 98554 -jet events from the data of the two years. They are sorted according to the values of our two observables into a $20 \times 20$ matrix.

To determine the influence of the DELPHI detector, tuned matrix element and parton shower simulations of 1991 (7098 4-jets and 8320 4-jets respectively) and special matrixelement simulations based on 4-parton final states (1990: 28422 4-jets and 1991: 11645 4-jets) have all been studied with full simulation of the DELPHI detector. For the ME simulation in JETSET 7.2, $y_{\text {cut }}$, the minimum invariant mass-squared of any two partons scaled by $E_{c m s}^{2}$, was set to 0.010 . This $y_{\text {cut }}$ corresponds to $m_{\text {eff }}=9.1 \mathrm{GeV}$ and is below the cut imposed by LUCLUS.

These subsets have been generated in slightly different conditions, but the detector corrections calculated from each subset are consistent with each other; the comparison of the correction matrix from simulation of 1990 with the combined one from the simulations of 1991 gives $\chi^{2} /$ dof $=430 / 399$. The direct comparison of the 42794 -jets from the data of 1990 with the 98554 -jets from the data of 1991 gives $\chi^{2} /$ dof $=390 / 399$. Thus there is no significant change in the response of charged particles as far as this analysis is concerned and the analysis can be performed with the combined data and simulations of 1990 and 1991. Adding up all simulations gives 55485 4-jet events.

Consistency between the simulated and the real 4-jet samples was first checked by comparing their thrust distributions; then with the event plane and axis defined by the sphericity tensor, the transverse particle momenta within and perpendicular to the event plane were examined. Finally, for each of the jets 1 to 4 , the distribution of the jet momentum, of the charged multiplicity of the jet and of the transverse and longitudinal particle momenta relative to the jet axis were examined. The average values agree typically within about $3 \%$.

The same global and track cuts were applied to generator level events (i.e. without detector simulation) as those described earlier for data and full detector level simulated 
events. The accuracy with which the observables $\left|\cos \theta_{N R}^{*}\right|$ and $\cos \alpha_{34}$ are measured is shown in fig. 4 for generated events and for events passed through the full detector simulation; the differences in $\left|\cos \theta_{N R}^{*}\right|$ and in $\cos \alpha_{34}$ at parton- and hadron-jet level are plotted. The resolution curves for generated events and for events after full detector simulation are practically the same. This shows that the uncertainty in the jet angles is dominated by the cluster algorithm, and that the jet angles are altered very little by the small loss of charged particles in the central part of the DELPHI detector. This similarity of the resolution curves permits the correction of the data to the physical distribution and vice versa without unfolding for additional smearing.

Figs. 5a and 5b show the distributions in the analysis observables of the 4-jet events after full simulation and at the generator level respectively. The comparison of figs. 5a and $5 \mathrm{~b}$ gives the $20 \times 20$ correction matrix in fig. $5 \mathrm{c}$ for the relation between these two distributions. For the analysis the three rows with $\cos \alpha_{34} \geq 0.7$ and 10 triangular arranged bins in the corner around $\left|\cos \theta_{N R}^{*}\right|=1, \cos \alpha_{34}=-1$, where the acceptance decreases due to the $d_{\text {join }}$-cut on the jet distances, have been omitted. The data contain 13057 4-jet events in the 330 remaining bins.

A smooth correction function $\eta$ was obtained by fitting the correction matrix with a 4th-order polynomial in the two observables following the ansatz:

\section{$(\text { Prediction for full simulation })_{\mathbf{1 , m}}=(\text { Generator level distribution })_{\mathbf{1}, \mathrm{m}} * \eta_{\mathbf{1}, \mathrm{m}}$}

The matrix representation $\eta_{\mathbf{l}, \mathbf{m}}$ of the correction function is shown in fig. 5d. Due to the same global and track cuts in both samples the correction function is rather flat. It varies only between 0.95 and 1.07 for the accepted range.

\section{Analysis}

\subsection{Fit of the Casimir-factors}

The 4-jet events are sorted into a $20 \times 20$ matrix according to their values of $\left|\cos \theta_{N R}^{*}\right|$ and $\cos \alpha_{34}$. The predictions are fitted to a selected range of these $20 \times 20$ bins with a maximum likelihood method. Poisson distributions for the likelihood factor are used in each bin.

The basis of the fit are two-dimensional reference distributions $\mathbf{A}_{\mathbf{l}, \mathbf{m}}, \mathbf{B}_{\mathbf{l}, \mathbf{m}}, \mathbf{C}_{\mathbf{l}, \mathbf{m}}, \mathbf{D}_{\mathbf{l}, \mathbf{m}}$, $\mathbf{E}_{\mathbf{l}, \mathbf{m}}$ in the form of $20 \times 20$ matrices in $\left|\cos \theta_{N R}^{*}\right|$ and $\cos \alpha_{34}$ for the classes $\mathrm{A}, \mathrm{B}, \mathrm{C}$, D, E. Events of class B and E cannot be generated directly since for some values of the kinematical variables their contribution to the cross section becomes negative. The combined contributions of $\mathrm{A}, \mathrm{B}$ and also of $\mathrm{D}, \mathrm{E}$ are positive. Class $\mathrm{C}$ is extracted directly from the QCD-events. The classes A,B and D,E contribute with different factors in QCD and the Abelian theory and this allows one to separate A from B and D from E. With the QCD values of the Casimir-factors, 2.5 million 4-jets events were generated. An additional 1.1 million 4-jets events have been generated in the Abelian theory with its different Casimir-factors.

The very large statistics of these generator events gives reasonably smooth reference distributions and there is no problem with a bias in this analysis. The two-dimensional 
reference distributions for the groups A, B, C, D, E are shown in figs. 6a. Projections of the distributions are given in figs. 6b. The relative contributions of the groups A, B, C, D, E are $35 \%,-6 \%, 64 \%, 7 \%,-0.2 \%$ for QCD and $26 \%, 34 \%, 0 \%, 39 \%, 1 \%$ for the Abelian theory. In $\theta_{N R}^{*}$, secondary $q \bar{q}$ events (class D) differ markedly from events with a pair of gluons, but triple-gluon events (class C) and double-bremsstrahlung events (classes A and B) look quite similar; they are distinguished by the second observable $\alpha_{34}$. From a combined fit of both variables, the contribution of all classes can be separated.

The theoretical prediction for the number of 4-jets from the generator of 4-parton events is given for each bin $1, \mathrm{~m}$ by:

$$
\begin{aligned}
\mathbf{T}_{\mathbf{l}, \mathbf{m}}= & \mathbf{N} * \alpha_{\mathbf{s}}^{2} * \mathbf{C}_{\mathbf{F}}^{2} * \\
& {\left[\mathbf{A}_{\mathbf{l}, \mathbf{m}}+\left(1-\frac{1}{2} \frac{\mathbf{C}_{\mathbf{A}}}{\mathbf{C}_{\mathbf{F}}}\right) \mathbf{B}_{\mathbf{l}, \mathbf{m}}+\frac{\mathbf{C}_{\mathbf{A}}}{\mathbf{C}_{\mathbf{F}}} \mathbf{C}_{\mathbf{l}, \mathbf{m}}+\frac{\mathbf{T}_{\mathbf{R}}}{\mathbf{C}_{\mathbf{F}}} \mathbf{D}_{\mathbf{l}, \mathbf{m}}+\left(1-\frac{1}{2} \frac{\mathbf{C}_{\mathbf{A}}}{\mathbf{C}_{\mathbf{F}}}\right) \mathbf{E}_{\mathbf{l}, \mathbf{m}}\right] }
\end{aligned}
$$

where $\mathbf{N}$ is the overall normalisation factor and $\mathbf{A}, \mathbf{B}, \mathbf{C}, \mathbf{D}, \mathbf{E}$ are the reference distributions from the Monte Carlo simulations described above. The distribution with which the data is compared is then

$$
\mathbf{P}_{\mathbf{1 , m}}=\left(\mathbf{T}_{\mathbf{l}, \mathbf{m}}+\mathbf{F}_{\mathbf{1}, \mathbf{m}}\right) * \eta_{\mathbf{1}, \mathbf{m}}
$$

$\mathbf{F}_{\mathbf{l}, \mathbf{m}}$ represents the background to the 4-jet events from fragmentation fluctuations of 3- and 2-parton events. Its integral contribution has been determined from the fullsimulation of detector effects with the complete QCD matrix element and amounts to $1.51 \pm 0.15 \%$ of the 4 -jet events. The shape is well determined by the high statistics of generated events, which contain 6009 background 4-jet events. The distributions are shown in fig $7 \mathrm{a}$ and $7 \mathrm{~b}$. This background has a sizeable influence as its shape is completely different from the reference distributions. For the extra errors introduced in the results due to the limited statistics in full detector simulation of this background, see section 5.4.

$\eta_{\mathbf{1}, \mathbf{m}}$ is the correction factor discussed before (see fig. $5 \mathrm{~d}$ ), which takes into account the influence of detector effects.

A maximum likelihood fit was then performed to the $\left|\cos \theta_{N R}^{*}\right| \mathrm{v} \cos \alpha_{34}$ distribution in terms of the three variables $X_{1}=\mathbf{N} * \alpha_{\mathbf{S}}^{2} * \mathbf{C}_{\mathbf{F}}^{2}, X_{2}=\frac{\mathrm{C}_{A}}{\mathrm{C}_{\mathrm{F}}}$, and $X_{3}=\frac{\mathbf{T}_{\mathrm{R}}}{\mathrm{C}_{\mathbf{F}}}$, using MINUIT [23] for maximisation. This was done simultaneously with the fit of the correction function. In this way, the influence of the finite statistics of the detector simulation on the result of $C_{A} / C_{F}$ and $T_{R} / C_{F}$ is included. A bin by bin correction using directly the unfitted correction matrix has also been tried and gives consistent results. In this case the influence of the finite statistics of the full simulation on the errors of the fit-parameters $C_{A} / C_{F}$ and $T_{R} / C_{F}$ has been taken into account by a global scaling factor deduced from the ratio of the number of 4 -jet events in full detector simulation to that of data. Fit results are given in tables 2 and 3 and discussed in section 5 . The quoted $\chi^{2}$ values have been calculated afterwards using the results of the likelihood fits. Fig. 8 shows the two-dimensional distributions in $\left|\cos \theta_{N R}^{*}\right|$ and $\cos \alpha_{34}$ for the data and for the result of the fit of the 330 bins with the 4th-order polynomial for the correction function. The bins which are not included in the fit are also shown. 


\subsection{Comparison of Parton Shower Model and Matrix Element Description}

The two-dimensional distribution in $\left|\cos \theta_{N R}^{*}\right|$ and $\cos \alpha_{34}$ from the data of 1991 (9855 4-jets) was also compared directly with that from full detector-simulation based on the parton-shower model, which is known to describe global event distributions well. In order to arrive at a clear distinction by $\chi^{2}$ for the shapes, $4 \times 4$ binning was performed. ME (7098 4-jets) and PS-simulations (8320 4-jets) are normalised to the same total number of events as contained in the data (9855 4-jets), and then the shapes are compared in fig. 9. ME-simulation shows a $\chi^{2} / d o f=13.36 / 15$, in very good agreement with the data. PS-simulation gives $\chi^{2} / d o f=45.77 / 15$, corresponding to a confidence level of $810^{-5}$. Clearly one needs the exact second order QCD ME to describe the 4-jet angular distributions. The leading log approximation used in the PS model is not sufficient.

\section{Systematic Errors}

\subsection{Parametrisation of the correction function}

Results are given in table 2 for the fit with bin by bin correction without smoothing, and for the combined fits with parametrisations of the correction function from a constant up to a 6 th order polynomial. From 3rd order on, the $\chi^{2} / d o f$ no longer improves and moreover the results for the physical variables $C_{A} / C_{F}$ and $T_{R} / C_{F}$ do not change and agree in their values with those obtained in the bin by bin correction. Thus there is no indication for a systematic shift as soon as one takes 3rd or higher order correction functions. The 4th-order result was chosen to be quoted.

\subsection{Influence of the bin selection}

To test the stability of the fit values, one row of bins or further triangular-arranged bins in one corner have been added or omitted. The results are given in table 3 , in each case for the bin by bin correction, and for the parametrisation by the 4th-order two-dimensional correction function. Also for this non-standard bin selection, results for other orders have been obtained. They are not shown as in all considered cases the results were stable starting from 3rd order. Typically the change for $C_{A} / C_{F}$ is below 0.06 and for $T_{R} / C_{F}$ below 0.3 . The largest change arises when the row $0.95 \leq\left|\cos \theta_{N R}^{*}\right| \leq 1.00$ is omitted. This is the row with the highest contents. Even then the changes of 0.15 and 0.4 respectively are less than the statistical errors of 0.30 and 0.7 . There is no indication of a significant systematic influence caused by different bin selections around the standard 330 accepted bins.

If all 400 bins are included, the $\chi^{2} /$ dof in the fits is worse. This is expected as reference distributions from the generator level and the full detector simulation disagree near the kinematic boundaries. 


\subsection{Different correction functions for the classes}

A universal correction function for detector effects has been used for the five reference distributions. It is not possible to determine the correction functions separately for the five classes, since the high statistics needed for the separation in the five classes cannot be achieved with full simulation of the detector. However separate corrections for $\mathrm{C}$ and the combined classes $\mathrm{AB}$ and DE as they appear in QCD can be deduced. As the contribution of class B is only $6 \%$ and that of $\mathrm{E}$ only $0.2 \%$ and the fit-result is near the QCD values, one gets a properly weighted estimate of the possible influence of separately determined correction functions. Extra correction functions, by which the universal correction function is independently multiplied, are determined from the comparison of fully simulated and generator distributions for these three contributions of double-bremsstrahlung, triple-gluon vertex, and secondary quark-antiquark production. The results for the extra linear correction functions are given in table 4, together with the resulting shift on the fit results of $C_{A} / C_{F}$ and $T_{R} / C_{F}$. The errors for these possible shifts have been determined by quadratic addition of the shifts induced by a change of each parameter by one standard deviation. None of these shifts is statistically significant. For the final result, the errors are increased by $50 \%$ since, instead of the separate classes A,B,D,E, only the combined classes AB and DE have been considered.

\subsection{Background from three- and two-parton events}

The background from 3 - and 2-parton events is inherently built into all the fits. If this background is 'switched off', there are changes of $\Delta\left(C_{A} / C_{F}\right)=+0.23$ and $\Delta\left(T_{R} / C_{F}\right)=-0.69$. This is despite the fact, that for the Matrix Element model with detector simulation, the background contribution is only $1.5 \%$ of the 4 -jet rate.

The uncertainty of these shifts is estimated by allowing an extra linear correction function with which the overall correction function is multiplied. The shape and the normalisation factor of the distribution from the 107 migrated events obtained from the full detector simulation (fig. 7a) is fitted to the one from generator level events (fig. 7b). The result is also shown in table 4 . This gives the resulting uncertainty due to the finite number of the events of this type available from full detector simulation. The errors have been increased by $50 \%$ for the final result to have a conservative estimate.

The 4-jet rate in the data is higher than that in the simulation. The difference can be attributed to 5-parton events in the data, with a soft or collinear parton which appear as 4-jet events. This effect is not present in the simulation, as the generator does not contain this higher order effect. The above shifts are therefore overestimated. The true shifts are obtained by scaling with the ratio $5.74 \% / 6.42 \%$ of the corresponding 4 -jet rates (see section 5.6) and are only +0.21 and -0.62 . Hence corrections of -0.02 for $C_{A} / C_{F}$ and +0.07 for $T_{R} / C_{F}$ have to be applied.

\subsection{Fragmentation}

The influence of the variation of the fragmentation parameters on the results has been studied by A. Seitz [24]. The five fragmentation parameters $a, b, \sigma_{q}, \epsilon_{c}, \epsilon_{b}$ have been chosen independently in a large range around their nominal values by a random generator. 
For each set events have been generated, and their distributions in rapidity ( 60 bins) and aplanarity (20 bins), which are sensitive to these parameters, were considered. Sets were accepted if the sum of the $\chi^{2}$ increase of these two distributions was below 60 . The events of the accepted sets were then considered as data, and fit values for $C_{A} / C_{F}$ and $T_{R} / C_{F}$ have been determined. The values of $C_{A} / C_{F}$ and $T_{R} / C_{F}$ for the sets which fulfilled the $\chi^{2}$-condition were compared with those from the events generated with the nominal fragmentation parameters. The rms of the shifts in the two parameters were 0.072 and 0.30 respectively.

In section 4.2 the Lund PS model was excluded. It is interesting to know if this conclusion remains the same if the parameters are varied. The 8320 -jet events from full detector simulation have been treated as 'data' in the fit-program for the Casimirfactors. The result is $C_{A} / C_{F}=2.21 \pm 0.32$ and $T_{R} / C_{F}=-2.0 \pm 0.6$. The negative value for $T_{R} / C_{F}$ is unphysical and 6 standard deviations away from the QCD-value of 1.875 . The variation of the fragmention parameters in ME-simulation has given rms of 0.3 for the shifts in $T_{R} / C_{F}$. This number should not depend much on the model. Even for a factor two bigger influence, the Lund PS simulation with variation of the fragmentation parameters is still inconsistent by 5 standard deviations with the QCD expectations.

\subsection{Influence of higher orders}

It is not possible to give a quantititative estimate of the influence of higher orders. The tree contributions in the next order $\alpha_{s}^{3}$ are available [25], but have not yet been incorporated into standard event generators. The internal loop-corrections to the 4parton final states have not yet been calculated. If QCD is the correct theory then the agreement of the results of this analysis with the QCD-values can be interpreted as an indication that the influence of the higher orders is relatively small.

The Parton Shower model contains higher orders in the leading log approximation (LLA); but it has been shown in sections 4.2 and 5.5 that this is not enough to describe the distributions which are sensitive to the triple-gluon vertex. Therefore it cannot be used for a quantitative estimate of the influence of higher orders. Higher orders manifest themselves in 5 -jet events. The data contain only $0.48 \%$ of 5 -jet events, the Parton Shower model $0.29 \%$, and the Matrix Element model $0.04 \%$. The last number gives the contribution from 4-parton events, appearing as 5-jets. This implies that in the data about $90 \%$ are real 5 jets (i.e. 5 -parton) events. The 4 -jet contributions are $6.4 \%, 5.6 \%$, and $5.7 \%$ respectively. That is, the 5 -jet contribution in the data amounts to $8 \%$ of the 4-jet rate. The rate is sizeable; this indicates that the contribution to 4-jets from 5-parton events with one unresolved parton pair might be sizeable too. But this contribution enters into the analysis only through the shape of the two dimensional angular distribution of its 4-jet events, which is probably not too different to that from genuine 4-parton events.

\subsection{Dependence on the $Y_{\text {cut }}$}

In the calculations of the parton cross-sections a $y_{c u t}$ is applied to handle the divergencies from soft and collinear gluons. On the parton level $y_{c u t}=0.01$ in the generator is below the cut imposed on the kinematical configuration by the value of $d_{\text {join }}$ in the LUCLUS cluster routine. When lowering the $y_{c u t}$ additional softer partons are produced, 
but after applying the cut criteria of the cluster routine on the parton configuration, exactly the same parton events survive. This independence on the $y_{c u t}$ is not perfect for the jets from the hadrons due to fluctuations in the fragmentation of the partons. The jet rates and the kinematical distributions still depend slightly on the choice of the $y_{\text {cut }}$.

Whereas the difference between data and simulation in the 4-jet rate can be considered as the unknown influence of higher orders, there is no such physical reason for a difference in the 3 -jet rates, as for the 3 -parton states the calculations contain also the contributions from the next order in $\alpha_{s}$. The observed difference of $1.1 \pm 0.2 \%$ in the 3 -jet rate of data and simulation has to be attributed to imperfect tuning. A way to get this aspect right is to change the $y_{c u t}$. A study at generator level (detector effects are expected to be small, see sect. 3) shows that the difference in the 3 -jet rate disappears for a reduction to $y_{\text {cut }}=0.0093$. As compared with the original $y_{\text {cut }}=0.01$, for $y_{\text {cut }}=0.0093$ the relative increase of the 4-parton cross section is $10 \%$, but that of the 4-jet rate is only $1.6 \pm 0.2$ $\%$.

The influence of this change in the $y_{c u t}$ on the result of the analysis has been determined by generating a large sample of events with the $y_{c u t}=0.0093$ and fitting these events as 'data' using in the fit the reference distributions with the nominal $y_{\text {cut }}=0.0100$. The results $C_{A} / C_{F}=2.36 \pm 0.06$ and $T_{R} / C_{F}=1.43 \pm 0.13$ are shifted with respect to the nominal values 2.25 and 1.875 . The shift were subtracted and the size of the shifts added as systematic errors.

\subsection{Influence of heavy quark masses}

The production of secondary heavy quark-antiquark pairs is suppressed. A separate study with the applied jet cut has shown that the different flavours are generated in the ratio $d: u: s: c: b=1: 1: 1:(0.98 \pm 0.02):(0.65 \pm 0.02)$. The effective number of active quark flavours is then $n_{f}^{e f f}=4.63 \pm 0.03$. This number is already built into the reference distributions at the generator level, which are used in the fit. Hence to reduce the result for $T_{R} / C_{F}$ to the value $T_{F} / C_{F}$ for one quark flavour, one has to divide by 5 .

\section{Discussion and Conclusions}

With the errors for the possible effects of separate correction functions, the corrections and errors for the background and $y_{c u t}$-dependence, and the uncertainties from the variation of the fragmentation parameters, the results are

$$
\begin{aligned}
& C_{A} / C_{F}=2.12 \pm 0.29(\text { stat. }) \pm 0.19(\text { corr. }) \pm 0.07(\text { fragm } .) \\
& T_{R} / C_{F}=2.31 \pm 0.66(\text { stat. }) \pm 0.60(\text { corr. }) \pm 0.30(\text { fragm } .)
\end{aligned}
$$

where $T_{R}=n_{f} T_{F}$. Using $T_{F} / C_{F}=N_{C} / N_{A}$, and $n_{f}=5$, and adding the errors in quadrature results in

$$
C_{A} / C_{F}=2.12 \pm 0.35 \quad \text { and } \quad N_{C} / N_{A}=0.46 \pm 0.19
$$


The measured variables $C_{A} / C_{F}$ and $N_{C} / N_{A}$ represent the ratios of the coupling strength of the triple-gluon vertex to that of gluon bremsstrahlung from a quark, and of the number of quark colours to the number of gluons. The plot with the contours for $68 \%$ and $95 \%$ confidence levels is given in fig. 10 .

The result for $C_{A} / C_{F}$ is in agreement with the value $9 / 4$ expected for QCD. The value for $N_{C} / N_{A}$ is consistent with the QCD value of $3 / 8$.

It is evident that the triple-gluon vertex must exist and that generally the number of quark colours has to be smaller than the number of gluons.

The expectations for various other gauge groups are given in fig. 10. The quarks are assumed to be in the fundamental representation and the gluons in the adjoint representation, except for $\mathrm{SU}(4)^{\prime}, \mathrm{SP}(4)^{\prime}$, and $\mathrm{SP}(6)^{\prime}$, which are examples with quarks in the next higher representation.

From table 1 it is evident that most groups in the plot are excluded by their inherent number of quark colours as this is directly determined experimentally from $R=\sigma\left(e^{+} e^{-} \rightarrow\right.$ hadrons $) / \sigma\left(e^{+} e^{-} \rightarrow \mu^{+} \mu^{-}\right)$and $\Gamma\left(\pi^{0} \rightarrow \gamma \gamma\right)$ as $N_{C}=3$. Apart from $\mathrm{SU}(3)$ and the ad hoc invented Abelian model U(1) 3 , only SO(3) has 3 colours for the quarks, but only 3 gluons in contrast to QCD which has 8 gluons. The result excludes also $\mathrm{SO}(3)$ as a candidate and allows only QCD with 8 gluons.

The previous analysis [15] of the data of 1989 and part of the data of 1990 gave $N_{C} / C_{F}=2.55 \pm 0.55$ (stat. $) \pm 0.4$ (fragm. + models $) \pm 0.2($ error in bias $)$ and $T_{R} / C_{F}=$ $0.1 \pm 2.4$. A preliminary analysis of the complete data of 1990 has been presented at the Geneva Conference 1991 [26]. The contribution of the triple-gluon vertex has now also been established by ALEPH [27]. They use as variables the five kinematical invariants. The shortcoming of this method is that, due to the many variables, they cannot correct directly for the efficiencies of the detector. Their results are $N_{C} / C_{F}=2.24 \pm 0.32($ stat. $) \pm$ 0.24 (syst.) and $T_{F} / C_{F}=0.58 \pm 0.17$ (stat.) \pm 0.23 (syst.).

In summary, it is found that the data require the existence of the triple-gluon vertex contribution in the QCD second order matrix element description. The parton shower version, which contains higher orders only in the leading-log approximation, does not describe quantitatively the distributions which are sensitive to the triple-gluon vertex. Other gauge groups with less than 8 gluons or more than 3 quark colours are excluded.

\section{Acknowledgements}

Our thanks go to K. Chetyrkin, R. Decker, and T. Sjöstrand for many helpful discussions. The physical interpretation of the results in the context of gauge groups was clarified by the contributions from M. Jezabek. We are greatly indebted to our technical collaborators and to the funding agencies for their support in building and operating the DELPHI detector, and to the members of the CERN-SL Division for the excellent performance of the LEP collider. 


\section{References}

[1] L. DiLella, Ann. Rev. Nucl. Part. Sci. 35 (1985) 107

[2] T.F. Walsh and P.M. Zerwas, Phys. Lett. 93B (1980) 53

[3] ARGUS Collaboration, H. Albrecht et al., Z. Physik C31 (1986) 181

[4] S. Bethke: Plenary talk at the XXVI International Conference on High Energy Physis, Dallas 1992

[5] K. J. F. Gaemers and J. A. M. Vermaseren, Z. Physik C7 (1980) 81

[6] J. G. Körner, G. Schierholz and J. Willrodt, Nucl. Phys. B185 (1981) 365

[7] O. Nachtmann and A. Reiter, Z. Physik C16 (1982) 45

[8] M. Bengtsson and P. M. Zerwas, Phys. Lett. 208B (1988) 306

[9] G. Rudolph, Physics at LEP, CERN 86-02 (1986) Vol. 2, 150

[10] AMY Collaboration, I. H. Park et al., Phys. Rev. Lett. 62 (1989) 1713

[11] OPAL Collaboration, M. Z. Akrawy et al., Z. Physik C49 (1991) 49

[12] L3 Collaboration, B. Adeva et al., Phys. Lett. 248B (1990) 227

[13] VENUS Collaboration, K. Abe et al., Phys. Rev. Lett. 66 (1991) 280

[14] A. Ali et al., Nucl. Phys. B167 (1980) 454

[15] DELPHI Collaboration, P. Abreu et al., Phys. Lett. 255B (1991) 466

[16] T. Hebbeker, Tests of Quantum Chromodynamics in Hadronic Decays of $Z^{0}$ Bosons Produced in $e^{+} e^{-}$Annihilations, Phys. Rep. 217 (1992) 69

[17] M. Jezabek, private communications

[18] R. K Ellis, D. A. Ross and A. E. Terrano, Nucl. Phys. B178 (1981) 421

[19] M. Bengtsson, Z. Physik C42 (1989) 75

[20] S. Bethke, A. Ricker and P.M. Zerwas, Heidelberg-Aachen preprint PITHA 90/14

[21] DELPHI Collaboration, P. Aarnio et al., Phys. Lett. 240B (1990) 271

[22] T. Sjöstrand, Computer Phys. Comm. 39 (1986) 347

T. Sjöstrand and M. Bengtsson, Computer Phys. Comm. 43 (1987) 367

We use JETSET version 7.2, November 1989.

LUCLUS is described in T. Sjöstrand, Computer Phys. Comm. 28 (1983) 229

[23] F. James and M.Roos, CERN library program D506 on GENLIB

[24] A. Seitz, Diploma-Thesis, Karlsruhe preprint IEKP-KA/92-5

[25] N.K. Falck, D. Graudenz, G.Kramer, DESY report DESY 89-027

K. Hagiwara and D. Zeppenfeld, Nucl. Phys. B313 (1989) 560

F.A. Berends, W.T. Giele and H. Kuijf, Nucl. Phys. B321 (1989) 39

[26] DELPHI 91-52 Phys 107: Contribution to the Geneva- 91 Lepton-Photon Conference T. Hebbeker: Plenary talk at that Conference

[27] ALEPH Collaboration, D. Decamp et al., Phys. Lett. 279B (1992) 411 


\section{Table 1:}

\section{Expectation for the Observables in Different Gauge Theories}

The quarks are assumed to be in the fundamental representation and the gluons in the adjoint representation, except for the lines marked with $*$ where the quarks are in the next higher representation of this gauge group.

$N_{C} / N_{A}=$ ratio of quark colours to number of gluons

$C_{A} / C_{F}=$ ratio of coupling strength of triple-gluon vertex to gluon-bremstrahlung off quarks

\begin{tabular}{|c|c|c|c|c|}
\hline Group & Gluons: & Quarks: & $N_{C} / N_{A}$ & $C_{A} / C_{F}$ \\
\hline \hline $\mathrm{SU}(\mathrm{n})$ & $n^{2}-1$ & $\mathrm{n}$ & $\frac{n}{n^{2}-1}$ & $\frac{2 n^{2}}{n^{2}-1}$ \\
\hline$*$ & $n^{2}-1$ & $\frac{n(n-1)}{2}$ & $\frac{n}{2(n+1)}$ & $\frac{n^{2}}{(n+1)(n-2)}$ \\
\hline $\mathrm{SO}(\mathrm{n})$ & $\frac{n(n-1)}{2}$ & $\mathrm{n}$ & $\frac{2}{n-1}$ & $2-N_{C} / N_{A}$ \\
\hline $\mathrm{Sp}(2 \mathrm{n})$ & $n(2 n+1)$ & $2 \mathrm{n}$ & $\frac{2}{2 n+1}$ & $2+N_{C} / N_{A}$ \\
\hline$*$ & $n(2 n+1)$ & $2 n^{2}-n-1$ & $1-1 / n$ & $2-N_{C} / N_{A}$ \\
\hline$G_{2}$ & 14 & 7 & $1 / 2$ & 2 \\
\hline$F_{4}$ & 52 & 26 & $1 / 2$ & $3 / 2$ \\
\hline$E_{6}$ & 78 & 27 & $9 / 26$ & $18 / 13$ \\
\hline$E_{7}$ & 133 & 56 & $8 / 19$ & $24 / 19$ \\
\hline$E_{8}$ & 248 & 248 & 1 & 1 \\
\hline $\begin{array}{c}\mathrm{U}(1)_{3} \\
\mathrm{Abelian}\end{array}$ & 1 & 3 & 3 & 0 \\
\hline $\begin{array}{c}\mathrm{U}(1) \\
\text { QED-like }\end{array}$ & 1 & 1 & 1 & 0 \\
\hline
\end{tabular}




\section{Table 2:}

Results of the simultaneous fits of correction-function and data $\chi^{2} /$ dof after the likelihood fit is given for:

comb. = combined contribution from data and correction function corfun $=$ contribution from correction function alone data $=$ contribution from data alone $\cos \alpha_{34}<0.7$ and 10 triangular arranged bins omitted in corner $1,-1$ (330 bins used in fit, 'Minos-fit' errors are given) $\left(C_{A} / C_{F}, T_{R} / C_{F}\right.$ correlation coefficient is -0.30 for the 4th-order fit)

\begin{tabular}{||l||l|||||c|||c|c|c||}
\hline correction & $\begin{array}{c}\chi^{2} / d o f \\
\text { comb. }\end{array}$ & $\begin{array}{c}\chi^{2} / d o f \\
\text { corfun }\end{array}$ & $\begin{array}{c}\chi^{2} / d o f \\
\text { data }\end{array}$ & $C_{A} / C_{F}$ & $T_{R} / C_{F}$ \\
\hline bin by bin & & & 1.141 & $2.20_{-.29}^{+.30}$ & $1.95_{-.65}^{+.67}$ \\
\hline const & 1.109 & 1.157 & 1.060 & $2.09_{-.26}^{+.26}$ & $1.79_{-.57}^{+.59}$ \\
linear & 1.100 & 1.134 & 1.065 & $2.38_{-.28}^{+.29}$ & $1.70_{-.63}^{+.65}$ \\
quadratic & 1.099 & 1.132 & 1.066 & $2.26_{-.29}^{+.29}$ & $1.81_{-.64}^{+.67}$ \\
cubic & 1.093 & 1.102 & 1.085 & $2.21_{-.29}^{+.29}$ & $1.88_{-.65}^{+.67}$ \\
\hline 4th-order & 1.092 & 1.101 & 1.084 & $2.21_{-.29}^{+.29}$ & $1.93_{-.65}^{+.68}$ \\
\hline 5th-order & 1.091 & 1.096 & 1.085 & $2.23_{-.29}^{+.30}$ & $1.93_{-.65}^{+.67}$ \\
6th-order & 1.094 & 1.108 & 1.082 & $2.23_{-.29}^{+.30}$ & $1.93_{-.65}^{+.67}$ \\
\hline
\end{tabular}




\section{Table 3:}

As table 2, but different cuts at edges or in corner 1,-1

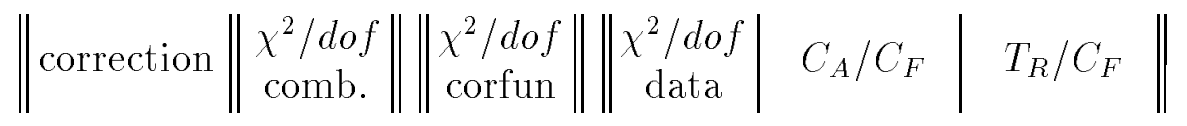

\begin{tabular}{|c|c|c|c|c|}
\hline $\operatorname{sic} \alpha_{34}<t$ & & & & \\
\hline $\begin{array}{l}\text { bin by bin } \\
4 \text { th-order }\end{array}$ & 1.107 & 1.135 & $\begin{array}{l}1.139 \\
1.080\end{array}$ & \begin{tabular}{l|l}
$2.15 \pm .31$ & $2.08 \pm .67$ \\
$2.16 \pm .31$ & $2.07 \pm .68$
\end{tabular} \\
\hline
\end{tabular}

\begin{tabular}{|c|c|c|c|c|c|}
\hline $\begin{array}{l}\text { bin by bin } \\
4 \text { th-order }\end{array}$ & 1.097 & 1.098 & $\begin{array}{l}1.147 \\
1.096\end{array}$ & $\begin{array}{l}2.26 \pm .28 \\
2.29 \pm .28\end{array}$ & $\begin{array}{l}1.88 \pm .66 \\
1.87 \pm .66\end{array}$ \\
\hline
\end{tabular}

15 bins omitted in corner (325 bins)

\begin{tabular}{||l||l|||l||l|l|l||}
\hline bin by bin & & & 1.141 & $2.26 \pm .30$ & $2.22 \pm .70$ \\
4th-order & 1.086 & 1.104 & 1.068 & $2.27 \pm .30$ & $2.22 \pm .70$ \\
\hline
\end{tabular}

6 bins omitted in corner (334 bins)

\begin{tabular}{||l||l||l|||l|l|l||}
\hline bin by bin & & & 1.159 & $2.12 \pm .29$ & $1.70 \pm .64$ \\
4th-order & 1.099 & 1.094 & 1.103 & $2.14 \pm .28$ & $1.68 \pm .63$ \\
\hline
\end{tabular}

\begin{tabular}{|c|c|c|c|c|c|}
\hline \multicolumn{6}{|c|}{$-0.9<\cos \alpha_{34}<0.7 \quad(314$ bins $)$} \\
\hline $\begin{array}{l}\text { bin by bin } \\
\text { 4th-order }\end{array}$ & 1.099 & 1.102 & $\begin{array}{l}1.146 \\
1.096\end{array}$ & $\begin{array}{l}2.17 \pm .30 \\
2.18 \pm .30\end{array}$ & \\
\hline
\end{tabular}

\begin{tabular}{|c|c|c|c|c|}
\hline $\operatorname{bin} 1$ & & & 1.109 & \begin{tabular}{|l|l|}
$2.06 \pm .32$ & $2.34 \pm .76$
\end{tabular} \\
\hline 4th-order & 1.077 & 1.104 & 1.051 & $2.06 \pm .31 \quad 2.34 \pm .76$ \\
\hline
\end{tabular}

nothing omitted (400 bins)

\begin{tabular}{||l||l|||l|||l|l|l||}
\hline bin by bin & & & 1.252 & $2.04 \pm .26$ & $1.16 \pm .58$ \\
4th-order & 1.163 & 1.158 & 1.168 & $2.09 \pm .26$ & $1.14 \pm .59$ \\
\hline const & 1.193 & 1.276 & 1.109 & $1.97 \pm .23$ & $1.55 \pm .54$ \\
\hline
\end{tabular}


Table 4:

Shifts induced on $C_{A} / C_{F}$ and $T_{R} / C_{F}$ by allowing deviations from the general correction function

$\mathrm{N}, \mathrm{X}, \mathrm{Y}$ are the fit results for the normalisation factor and slopes in $\left|\cos \theta_{N R}^{*}\right|$ and $\cos \alpha_{34}$ for extra linear correction functions, which are superimposed on the general 4th order correction function.

\begin{tabular}{||l||c|r|r||r|r||}
\hline Used for & $\mathrm{N}$ & \multicolumn{1}{c|}{$\mathrm{X}$} & \multicolumn{1}{c||}{$\mathrm{Y}$} & $\Delta\left(C_{A} / C_{F}\right)$ & $\Delta\left(T_{R} / C_{F}\right)$ \\
\hline \hline $\mathrm{AB}$ & $1.028 \pm .012$ & $-.020 \pm .017$ & $.029 \pm .020$ & $-.046 \pm .074$ & $-.11 \pm .11$ \\
\hline $\mathrm{C}$ & $0.996 \pm .008$ & $.001 \pm .012$ & $-.009 \pm .014$ & $-.022 \pm .031$ & $-.13 \pm .13$ \\
\hline $\mathrm{DE}$ & $0.977 \pm .020$ & $.044 \pm .035$ & $-.044 \pm .042$ & $.018 \pm .027$ & $.02 \pm .08$ \\
\hline Background & $1.11 \pm .20$ & $-.17 \pm .21$ & $-.10 \pm .23$ & $.020 \pm .057$ & $-.04 \pm .18$ \\
\hline
\end{tabular}

combined values

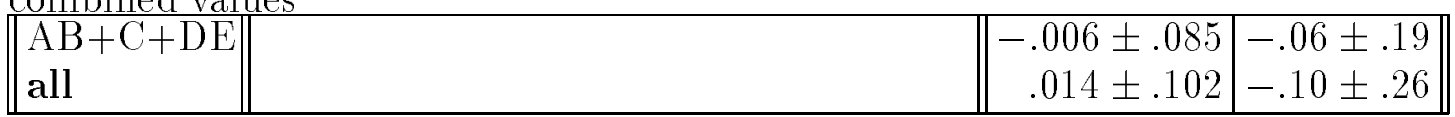




\section{Figure Captions}

Fig. 1 Diagrams that yield 4-parton final states

(a) Double-bremsstrahlung

(b) Secondary $q \bar{q}$ production

(c) Triple-gluon vertex

Fig. 2 Definitions of the generalised Nachtmann-Reiter angle $\theta_{N R}^{*}$ in terms of the jet momentum vectors $\vec{p}_{j}$, and of the angle $\alpha_{34}$ used in this analysis to distinguish the triple-gluon vertex contribution from that due to double-bremsstrahlung (The index $\mathrm{j}$ increases with decreasing jet energy).

Fig. 3 Casimir factors for the fundamental couplings.

Diagrams (a) and (c) have the same topology; the coupling strengths are related to the numbers of quark colours $N_{C}$ and gluons $N_{A}$ by $T_{F} / C_{F}=N_{C} / N_{A}$.

Fig. 4 Comparison of generator level with full detector simulated events. Resolution for the observables:
(a) $\left|\cos \theta_{N R}^{*}\right|$
(b) $\cos \alpha_{34}$
$\square$ : after detector-simulation
+ : generated events

Fig. 5 Two-dimensional distributions in $\left|\cos \theta_{N R}^{*}\right|$ and $\cos \alpha_{34}$

(a) 4-jets after full detector simulation

(b) 4 -jets events at the generator level

(c) correction matrix for the 330 accepted bins from a bin-by-bin comparison of (a) and (b).

(d) correction function from a 4th-order two-dimensional polynomial fit of these 330 bins.

Fig. 6 Distributions of the 4-jet events after cuts from the classes A,B,C,D,E

(deduced from large statistics of generated events for QCD and Abelian model)

(a) two-dimensional

(b) projections on $\left|\cos \theta_{N R}^{*}\right|$ and $\cos \alpha_{34}$ (without class E)

Fig. 7 Background to 4-jet events from 3- and 2-parton events (not normalised)

(a) after full detector simulation

(b) for generated events

Fig. 8 Two-dimensional distributions in $\left|\cos \theta_{N R}^{*}\right|$ and $\cos \alpha_{34}$

(a) Data

(b) Fitted distribution

Fig. 9 PS vs ME: Comparison with 1991 data
(a) Data
(b) QCD-ME full detector simulation
(c) $\chi^{2}$ in each bin from comparison of data and QCD-ME
(d) QCD-PS full detector simulation
(e) $\chi^{2}$ in each bin from comparison of data and QCD-PS 
Fig. $1068 \%$ and $95 \% \mathrm{CL}$ contour plots for the measured variables $C_{A} / C_{F}$ and $N_{C} / N_{A}$, and expections from different gauge theories

$C_{A} / C_{F}=$ ratio of coupling strength of $g \rightarrow g g$ to $q \rightarrow q g$ $N_{C} / N_{A}=$ number of quark colours divided by the number of gluons It is evident that the triple-gluon vertex must exist and that generally the number of quark colours has to be smaller than the number of gluons.

Quarks are in the fundamental and gluons in the adjoint representation (except $\mathrm{SU}(4)^{\prime}, \mathrm{SP}(4)^{\prime}, \mathrm{SP}(6)^{\prime}$, where the quarks are in the next higher representation). Most groups have $N_{C} \neq 3$ (see table 1 ) and are already excluded by $N_{C}=3$ from $R=\sigma\left(e^{+} e^{-} \rightarrow\right.$ hadrons $) / \sigma\left(e^{+} e^{-} \rightarrow \mu^{+} \mu^{-}\right)$and $\Gamma\left(\pi^{0} \rightarrow \gamma \gamma\right) . \quad N_{C}=3$ only for the Abelian model $\mathrm{U}(1)_{3}, \mathrm{SO}(3)$, and QCD (larger symbols in the fig.). From the result of this analysis also $\mathrm{SO}(3)$ is excluded as a candidate. 

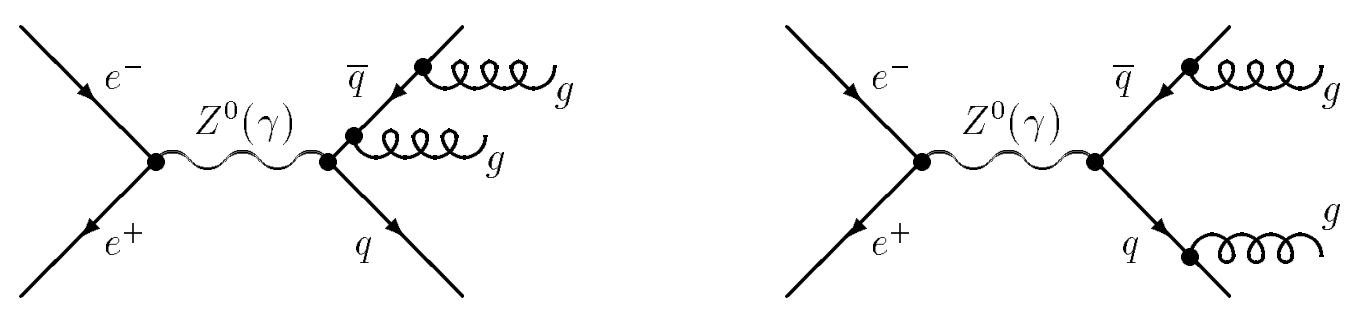

(a)

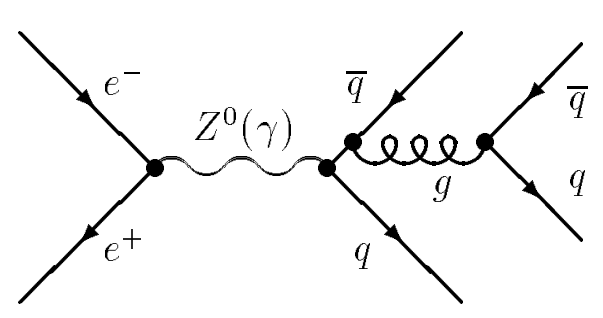

(b)

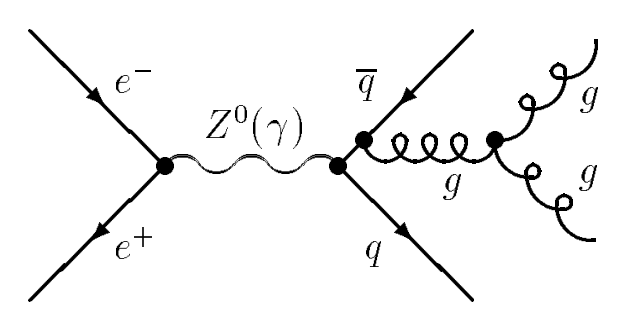

(c)

Fig. 1

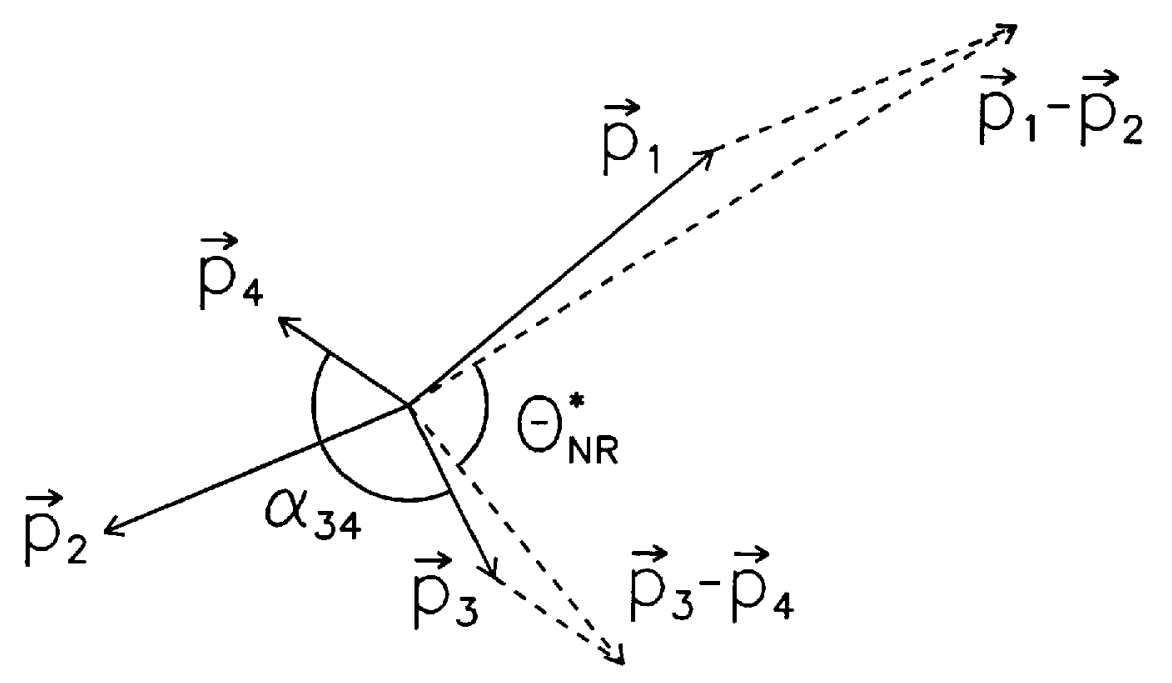

Fig. 2 
(a) $\left|i \longrightarrow g_{j}^{a}\right|^{2} \propto C_{F}$

(b) $\mid$ veetg $\left.\left.\right|^{b}\right|^{2} \propto C_{A}$

(c)

Fig. 3 

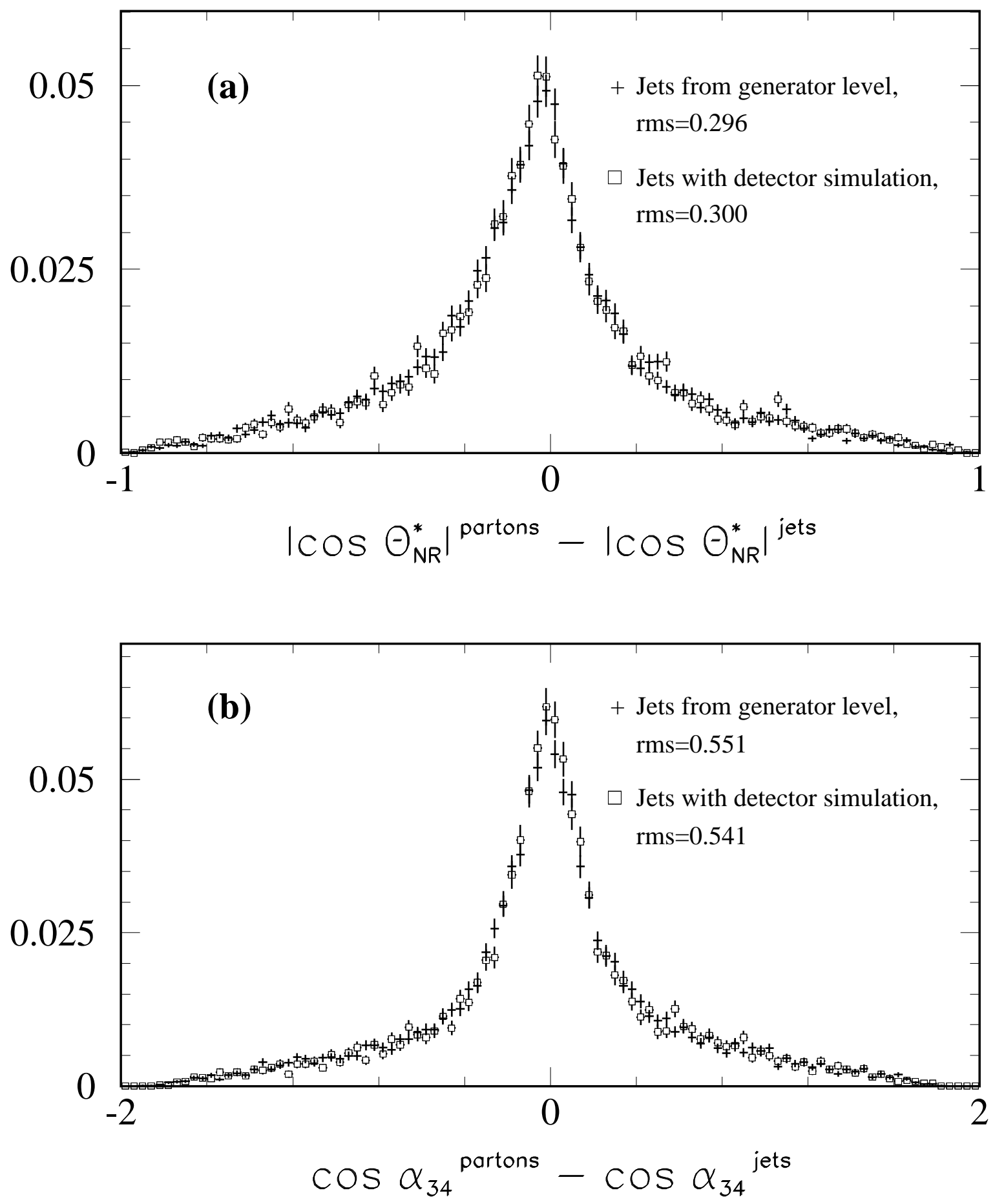

Fig. 4 


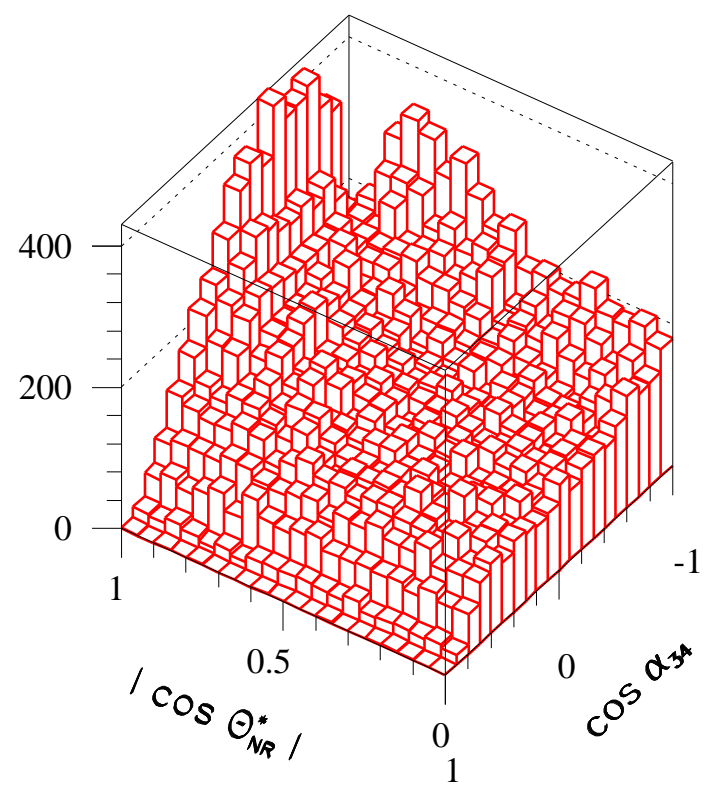

(a) DELPHI-Monte Carlo

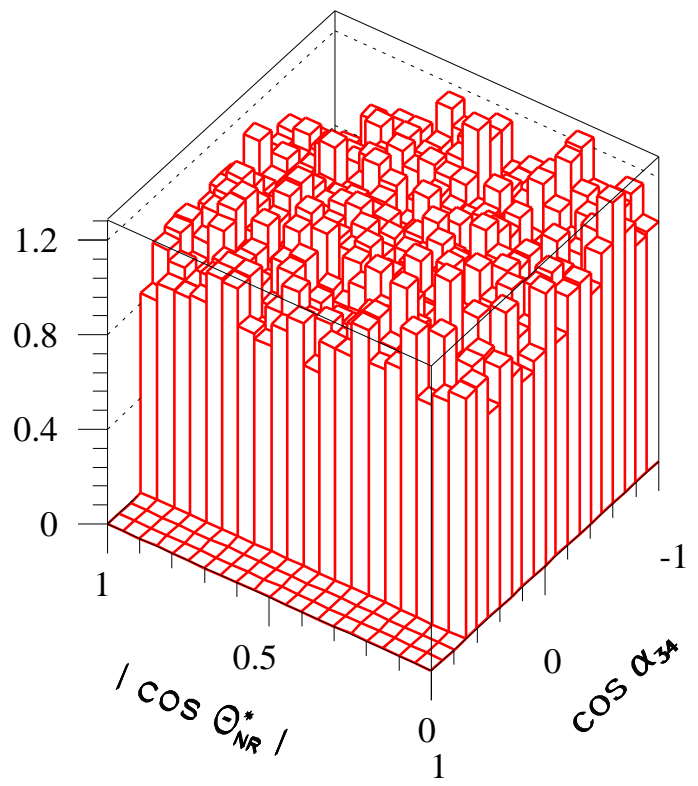

(c) correction matrix

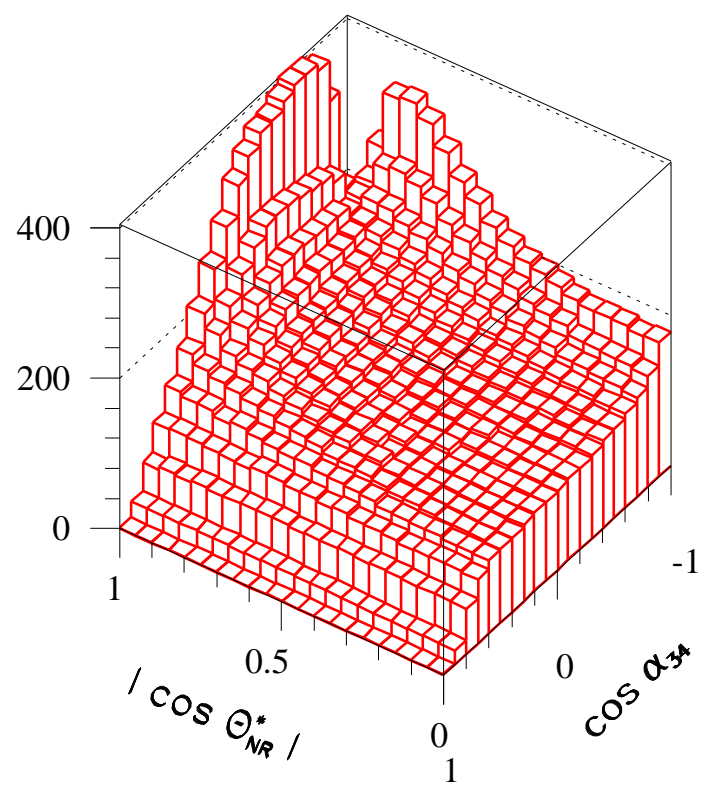

(b) Generator JETSET 7.2

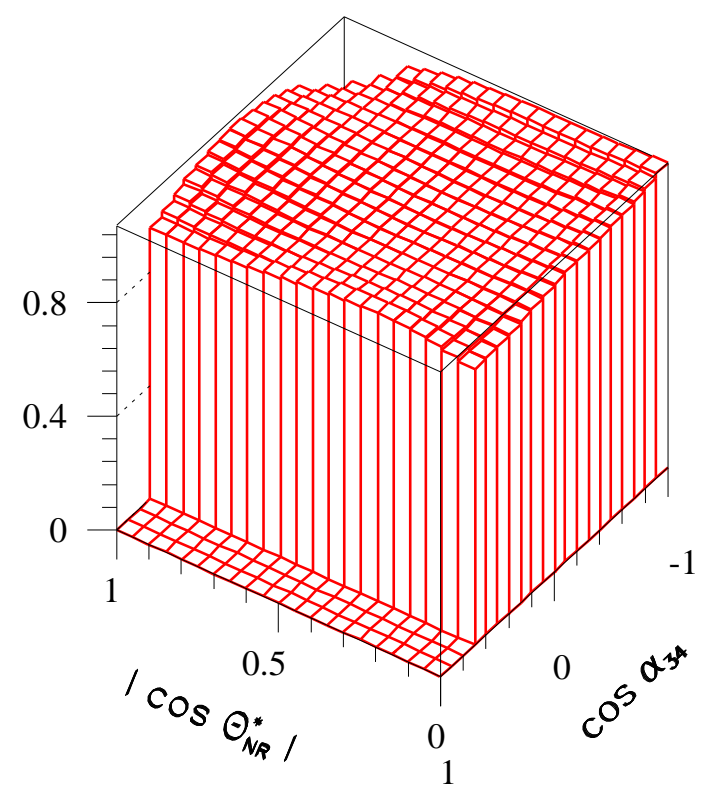

(d) correction function

Fig. 5 


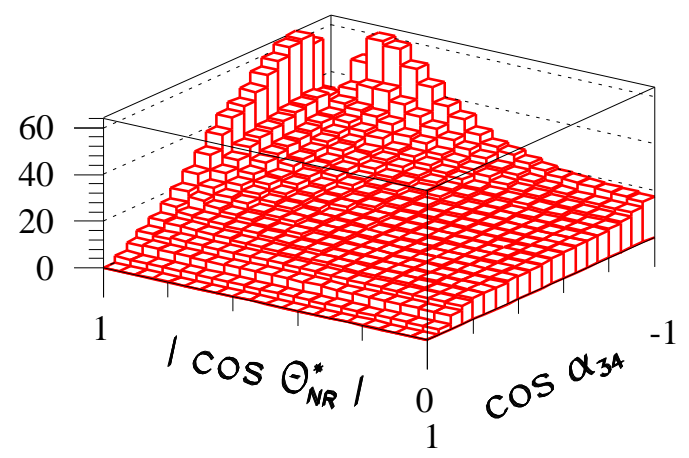

class A

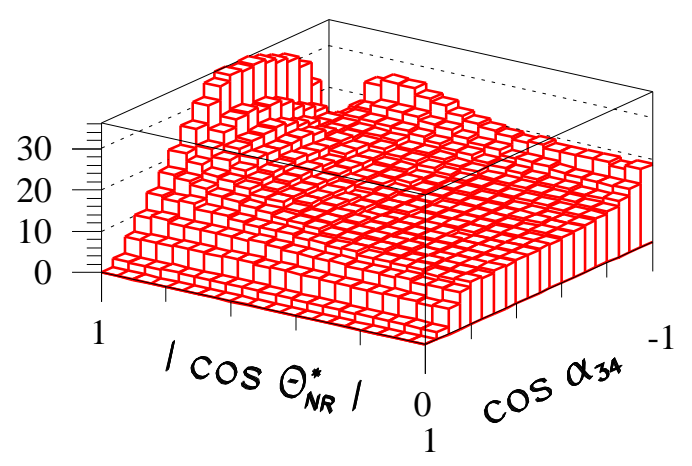

class $\mathbf{C}$

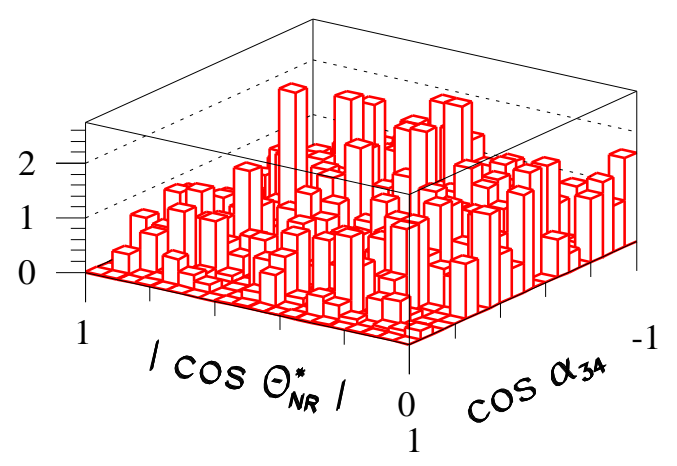

class $\mathbf{E}$

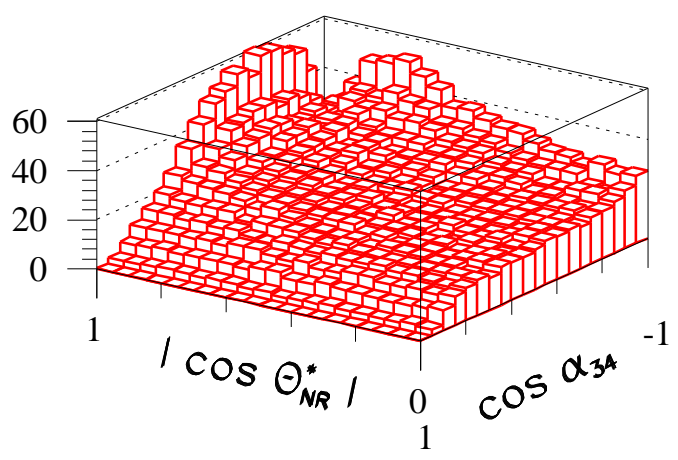

class B

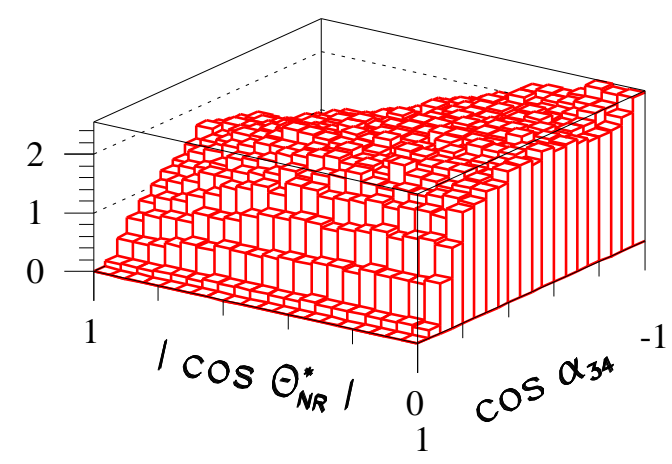

class D

Fig. 6a 

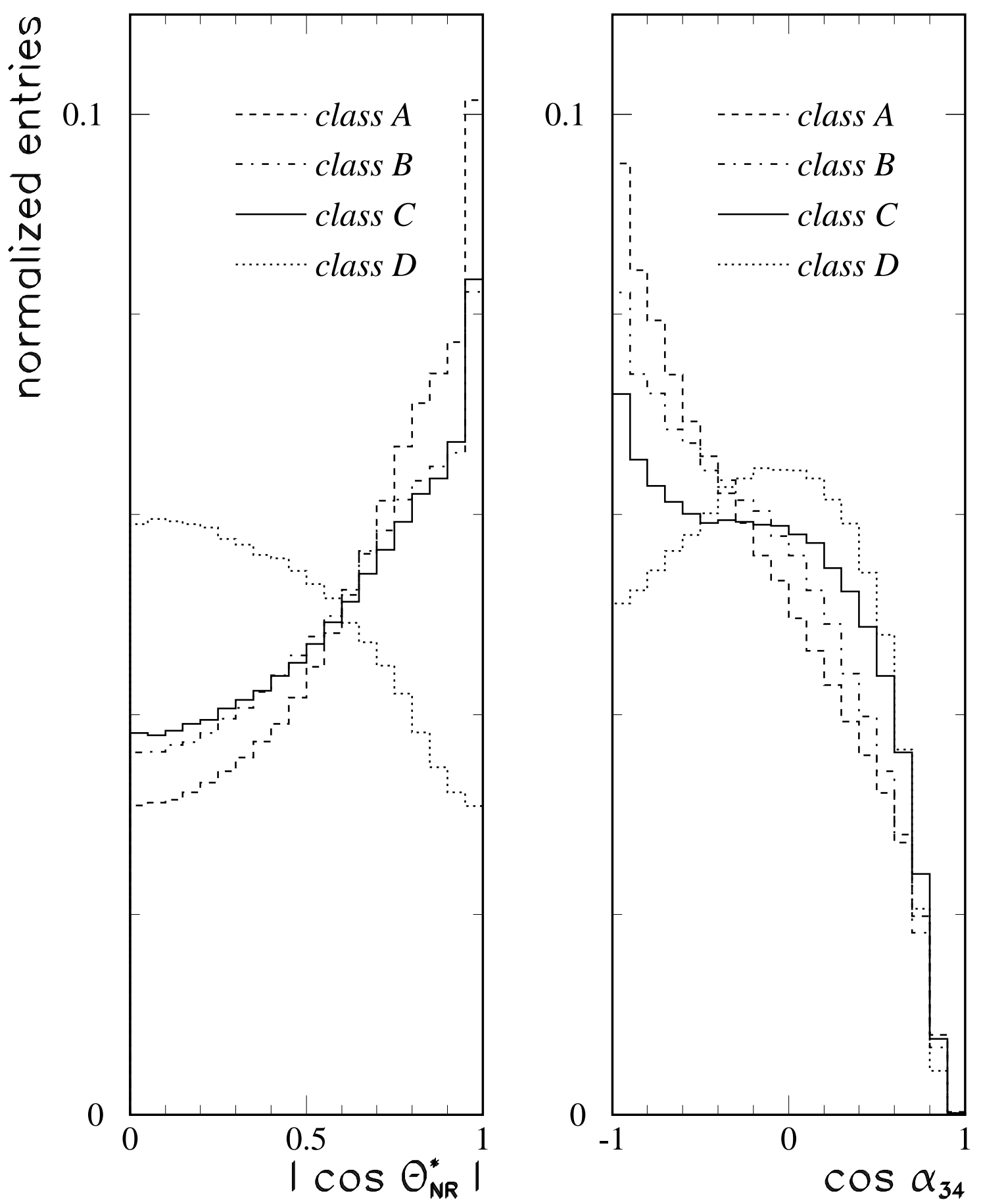

Fig. 6b 


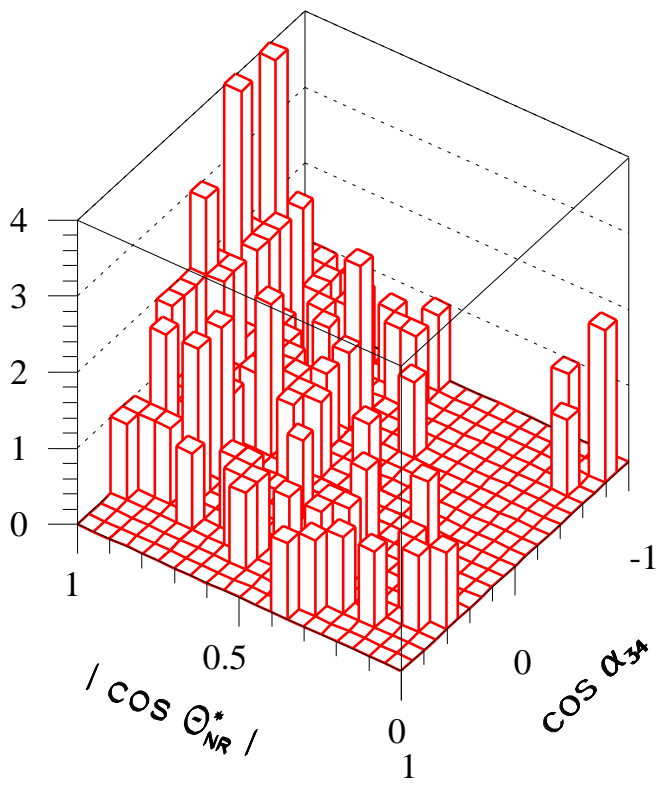

(a) background with detector simulation

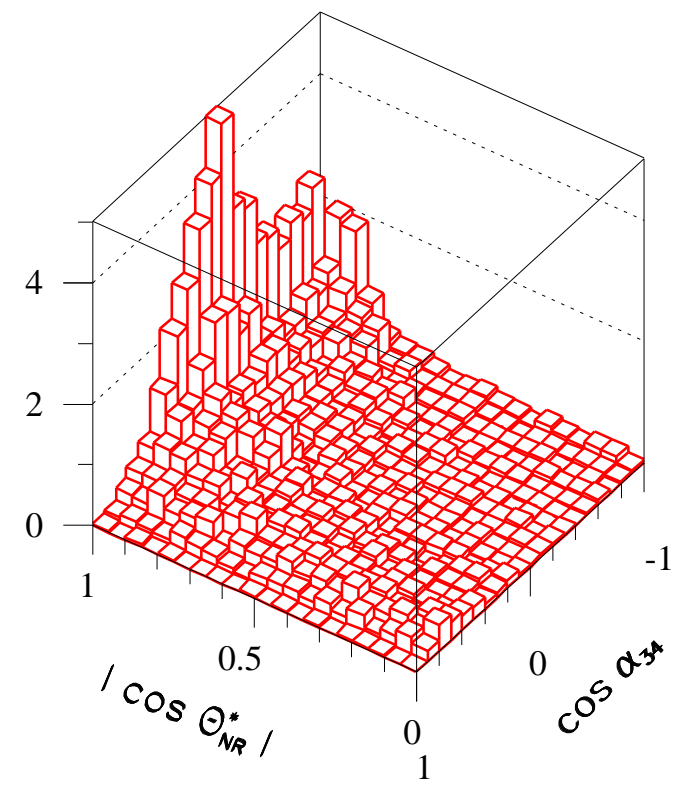

(b) background generator level events

Fig. 7

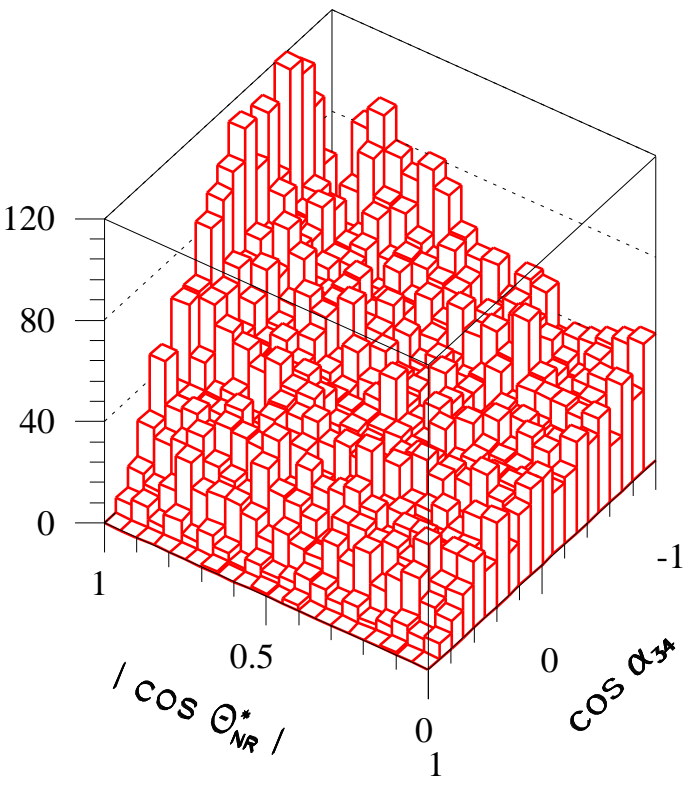

(a) DELPHI-Data

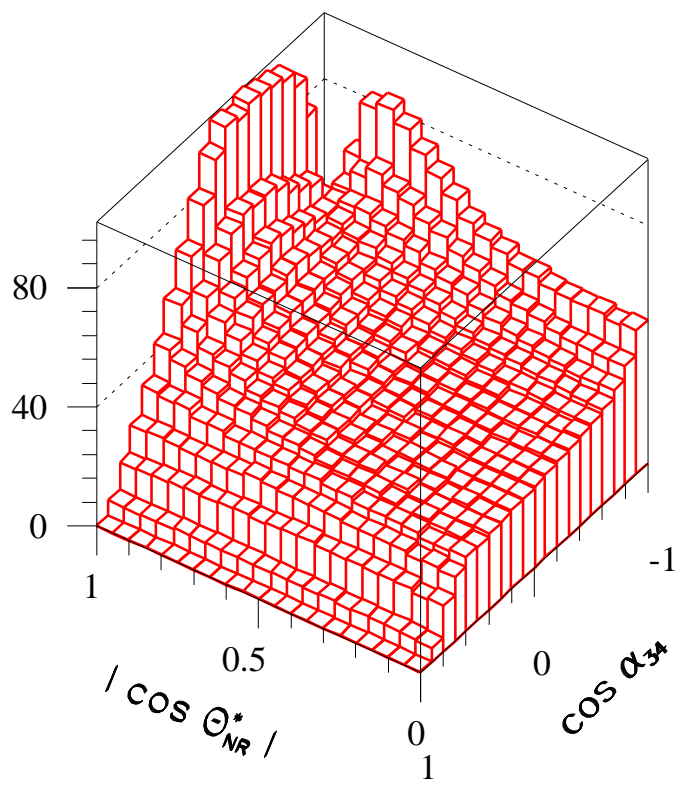

(b) fitted distribution

Fig. 8 
(a)

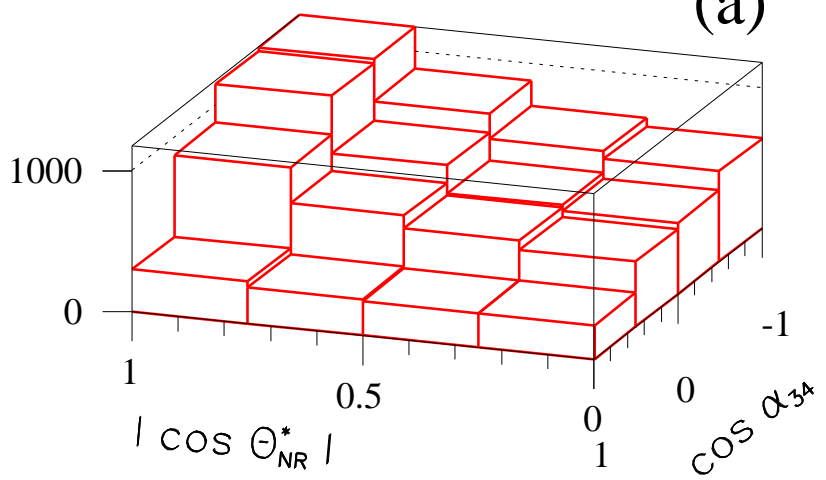

DELPHI - Data

(b)

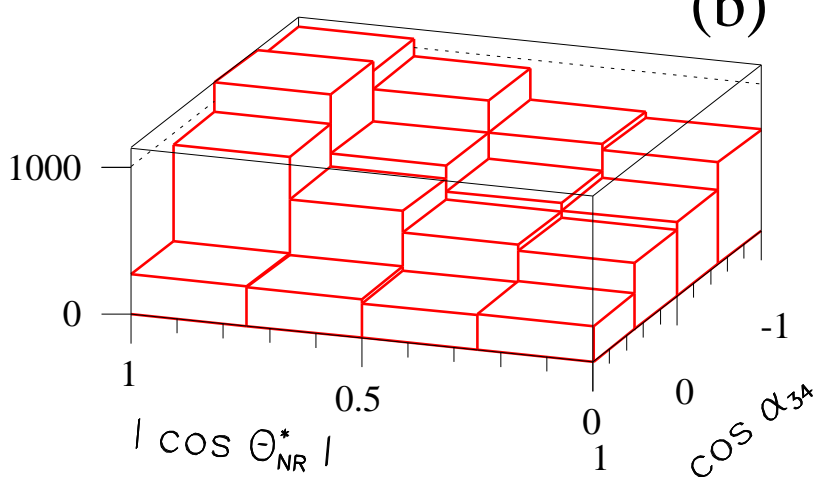

ME - simulation :

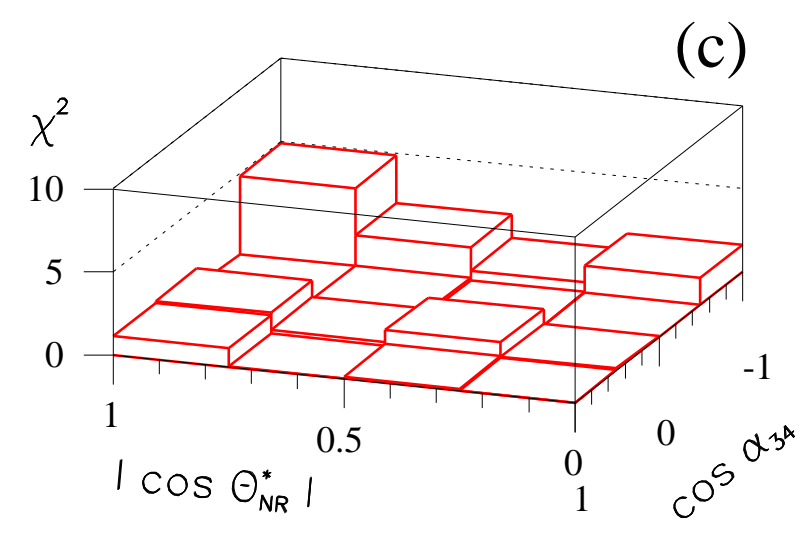

$\chi^{2}=13.4 \rightarrow C L=0.55$

(d)

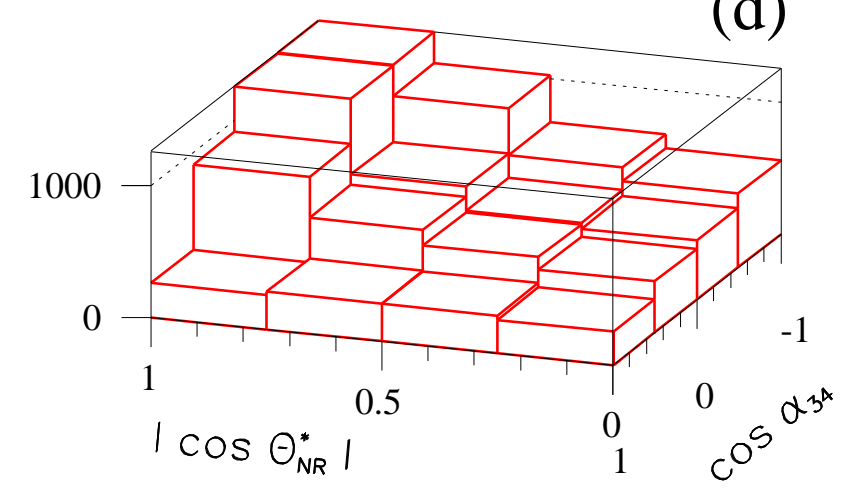

PS - simulation :

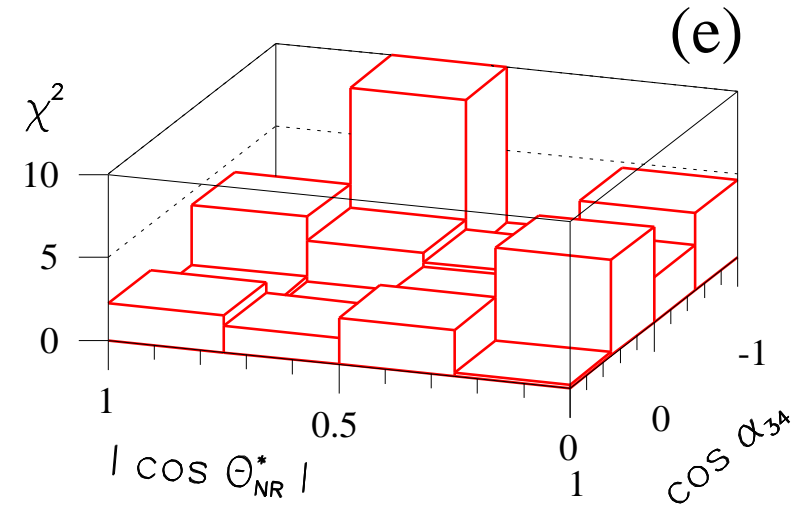

$\chi^{2}=45.8 \rightarrow C L=8 * 10^{-5}$

Fig. 9 


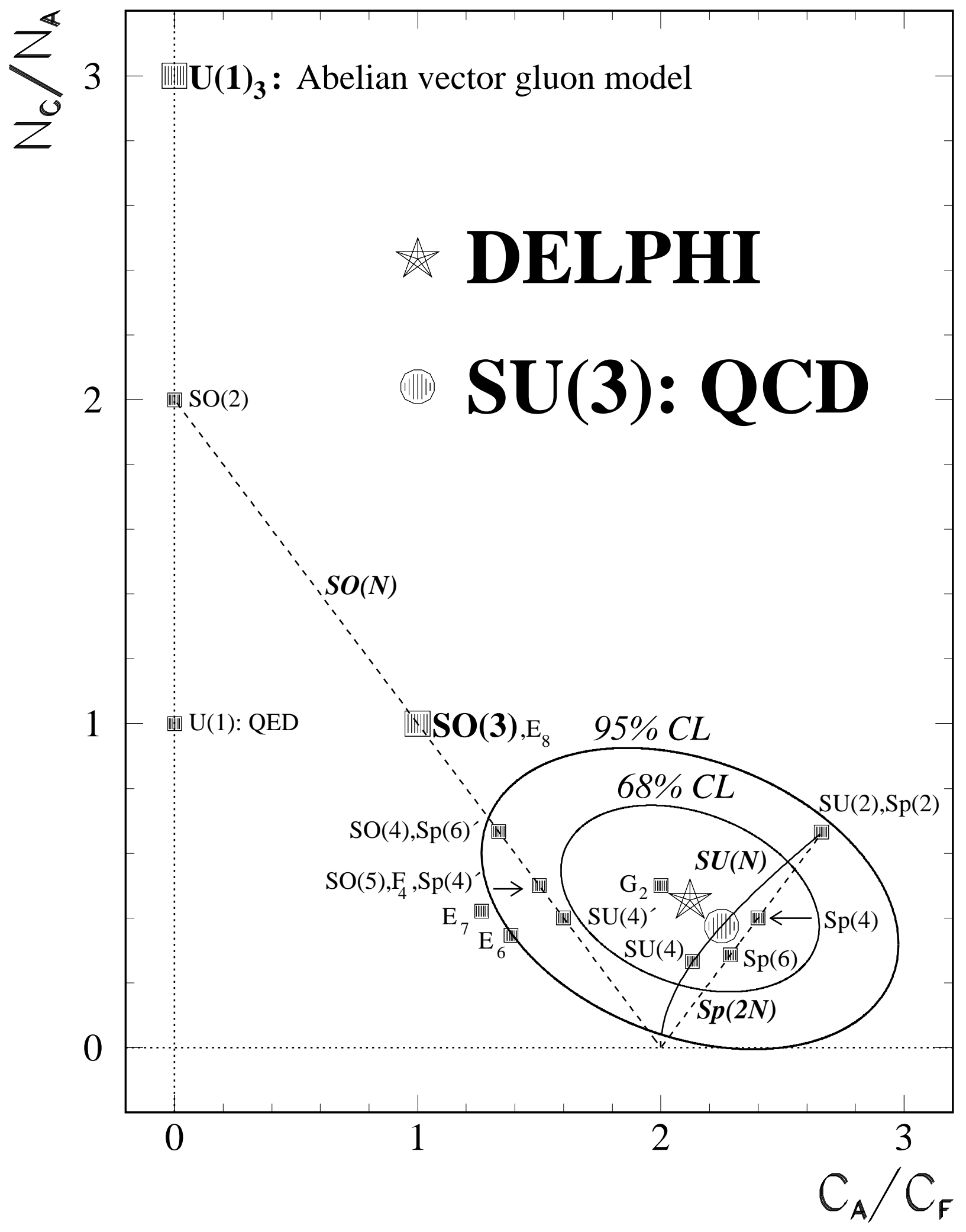

Fig. 10 